\title{
Two ten-billion-solar-mass black holes at the centres of giant elliptical galaxies
}

\author{
Nicholas J. McConnell ${ }^{1}$, Chung-Pei Ma ${ }^{1}$, Karl Gebhardt ${ }^{2}$, Shelley A. Wright ${ }^{1}$, Jeremy D. \\ TMurphy ${ }^{2}$, Tod R. Lauer ${ }^{3}$, James R. Graham ${ }^{1,4}$ and Douglas O. Richstone ${ }^{5}$
}

December 7, 2011

\section{U}

Department of Astronomy, University of California, Berkeley, California 94720, USA. ${ }^{2}$ Department of Astronomy, University of Texas, Austin, Texas 78712, USA. National Optical Astronomy Observatory, Tucson, Arizona 85726, USA. ${ }^{4}$ Dunlap 1 institute for Astronomy and Astrophysics, University of Toronto, Ontario, Canada. ${ }^{5}$ Department of Astronomy, University of Michigan, Ann Arbor, Michigan 48109, USA.

Observational work conducted over the last few decades indicates that all massive galaxies have supermassive black holes at their centres. Although the luminosities and brightness fluctuations of quasars in the early Universe suggest that some are powered by black holes with masses greater than 10 billion solar masses ${ }^{1 / 2}$, the remnants of these objects have not been found in the nearby Universe. The giant elliptical galaxy Messier 87 hosts the hitherto most massive known black thole, which has a mass of 6.3 billion solar masses 3 . Here we report that NGC 3842, the brightest galaxy in a cluster at a distance from Earth of 98 megaparsecs, has a central black hole with a mass of 9.7 billion solar masses, and that a black hole of comparable or greater onass is present in NGC 4889, the brightest galaxy in the Coma cluster (at a distance of 103 megaparsecs). These two black holes are significantly more massive than predicted by linearly extrapolating the widely-used correlations between black hole mass and the stellar velocity dispersion or bulge luminosity of the host galaxy [5.9. Although these correlations remain useful for predicting black hole masses in less massive elliptical galaxies, our measurements suggest that differont evolutionary processes influence the growth of the largest galaxies ând their black holes.

Empirical scaling relations between black hole mass $\left(M_{\mathrm{BH}}\right)$, galaxy ulge velocity dispersion $(\sigma)$, and luminosity $(L)$ are commonly used to esIntmate black hole masses, because for most galaxies we are unable to make a direct measurement. Estimates of the number density of black holes in a given mass range thus depend upon the empirically determined $M_{\mathrm{BH}}-\sigma$ and $M_{\mathrm{BH}}-L$ relations over an appropriate range of galaxy masses. Directly measuring $M_{\mathrm{BH}}$ from the kinematics of stars or gas in the vicinity of the black hole is particularly difficult at the highest galaxy masses, because massive galaxies are rare, their typical distances from Earth are large, and their central stellar densities are relatively low. The most massive galaxies are typically brightest cluster galaxies (BCGs), that is, giant ellipticals that reside near the centres of galaxy clusters.

We have obtained high-resolution, two-dimensional data of the lineof-sight stellar velocities in the central regions of NGC 3842 and NGC 4889 using integral field spectrographs at the Gemini North and Keck 2 telescopes, in Hawaii. The stellar luminosity distribution of each galaxy is provided by surface photometry from NASA's Hubble Space Telescope and ground-based telescopes 10,11 . NGC 3842 is the BCG of Abell 1367, a moderately rich galaxy cluster. NGC 4889 is the BCG of the Coma cluster (Abell 1656), one of the richest nearby galaxy clusters. We targeted these two galaxies because they have relatively high central surface brightnesses and lie at an accessible distance for direct measurements of $M_{\mathrm{BH}}$.

We measured the distribution of stellar velocities at 82 different locations in NGC 3842. The line-of-sight velocity dispersion in NGC 3842 is between 270 and $300 \mathrm{~km} \mathrm{~s}^{-1}$ at large galactocentric radii $(r)$ and rises in the central $0.7 \operatorname{arcsec}\left(r<330 \mathrm{pc}\right.$ ), peaking at $326 \mathrm{~km} \mathrm{~s}^{-1}$ (Figs 1 . 2. We determined the mass of the central black hole by constructing a series of orbit superposition models 12. Each model assumes a black hole mass, stellar mass-to-light ratio $\left(M_{\star} / L\right)$ and dark matter profile, and generates a library of time-averaged stellar orbits in the resulting gravitational potential. The model then fits a weighted combination of orbital line-of-sight velocities to the set of measured stellar velocity distributions. The goodness-of-fit statistic $\chi^{2}$ is computed as a function of the assumed values of $M_{\mathrm{BH}}$ and the stellar mass-to-light ratio. Using our best-fitting model dark matter halo, we measure a black hole mass of $9.7 \times 10^{9}$ solar masses $\left(M_{\odot}\right)$, with a $68 \%$ confidence interval of $7.2-12.7 \times 10^{9} M_{\odot}$. Models with no black hole are ruled out at the $99.996 \%$ confidence level $\left(\Delta \chi^{2}=17.1\right)$. We find the stellar mass-to-light ratio to equal $5.1 M_{\odot} / L_{\odot}$ in $R$ band $\left(L_{\odot}\right.$, solar luminosity), with a 68\% confidence interval of $\left.4.4 M_{\odot} / L_{\odot}-5.8 M_{\odot} / L_{\odot}\right)$.

We measured stellar velocity distributions at 63 locations in NGC 4889 and combined our measurements with published long-slit kinematics at larger radii 13 . The largest velocity dispersions in NGC 4889 are located across an extended region on the east side of the galaxy. The stellar orbits in our models are defined to be symmetric about the galaxy centre, so we constrain $M_{\mathrm{BH}}$ by running separate trials with velocity profiles from four quadrants of the galaxy. The best-fitting black hole masses from the four quadrants range from $9.8 \times 10^{9} M_{\odot}$ to $2.7 \times 10^{10} M_{\odot}$. All quadrants favor tangential orbits near the galaxy centre, which cause the line-of-sight velocity dispersion to decrease even as the internal three-dimensional velocity dispersion increases toward the black hole. Although no single model is consistent with all of the observed kinematic features in NGC 4889, we can define a confidence interval for $M_{\mathrm{BH}}$ by considering the most extreme confidence limits from the cumulative set of models. The corresponding $68 \%$ confidence interval is $(0.6-3.7) \times 10^{10} M_{\odot}$. We adopt a black hole mass of $2.1 \times 10^{10} M_{\odot}$, corresponding to the midpoint of this interval.

Figure 3 shows the $M_{\mathrm{BH}}-\sigma$ and $M_{\mathrm{BH}}-L$ relations, using data compiled from studies published before the end of August 2011, plus our measurements of NGC 3842 and NGC 4889. Tabulated data with references are provided as Supplementary Information. The most widely used form for both relations is a power law with a constant exponent. Straight lines in Fig. 3 show our fits to $M_{\mathrm{BH}}(\sigma)$ and $M_{\mathrm{BH}}(L)$. The relationship between $\sigma$ and $L$, however, flattens at high galaxy masses, and constantexponent power laws for the $M_{\mathrm{BH}}-\sigma$ and $M_{\mathrm{BH}}-L$ relations produce contradictory predictions for $M_{\mathrm{BH}}$ in this mass range 14 . Direct measurements of $M_{\mathrm{BH}}$ in higher mass galaxies will compel the revision of one or both of the $M_{\mathrm{BH}}-\sigma$ and $M_{\mathrm{BH}}-L$ relations. 
The average velocity dispersion in NGC 3842 is $270 \mathrm{~km} \mathrm{~s}^{-1}$, measured outside the black hole radius of influence $(1.2 \operatorname{arcsec}$ or $570 \mathrm{pc})$ and inside the two-dimensional half-light radius (38 $\operatorname{arcsec}$ or $18 \mathrm{kpc}$ ). Although NGC 3842 hosts a black hole more massive than any previously detected, its average dispersion ranks only fourteenth among 65 galaxies with direct measurements of $M_{\mathrm{BH}}$. Its luminosity ranks fifth in this sample of galaxies and is exceeded only by other BCGs. On the basis of $\sigma$ and $L$ for NGC 3842, our revised $M_{\mathrm{BH}}-\sigma$ and $M_{\mathrm{BH}}-L$ relations predict $M_{\mathrm{BH}}=9.1 \times 10^{8} M_{\odot}$ and $2.5 \times 10^{9} M_{\odot}$, respectively. Similarly, for NGC 4889 the respective predictions are $3.3 \times 10^{9} M_{\odot}$ and $4.5 \times 10^{9} M_{\odot}$. These predictions are smaller than our direct measurements of $M_{\mathrm{BH}}$, by 1.6-4.6 times the 1-s.d. scatter in the $M_{\mathrm{BH}}-\sigma$ and $M_{\mathrm{BH}}-L$ relations 9. Four measurements of $M_{\mathrm{BH}}$ in BCGs existed before this work. Two measurements based on gas dynamics and one based on stellar dynamics all lie within 1.2 s.d. of our revised fits to the $M_{\mathrm{BH}}-\sigma$ and $M_{\mathrm{BH}}-L$ relations 15,16 . Yet the measurement of $M_{\mathrm{BH}}$ in NGC 1316, the BCG of the Fornax cluster, is 3.4 s.d. less than that predicted by our $M_{\mathrm{BH}}-L$ relation ${ }^{17}$. The high scatter indicated by this collection of measurements reveals large uncertainties in the standard practice of using galacitc $\sigma$ or $L$ as a proxy for the central black hole mass in giant elliptical galaxies and their predecessors.

Several BCGs within $200 \mathrm{Mpc}$ of Earth are at least twice as luminous as NGC 3842, and three times as luminous as M87, which hosted the most massive black hole known before this work. In spite of their extreme luminosities, BCGs have velocity dispersions similar to those of the most massive field elliptical galaxies. Yet the most massive black holes are found predominantly in BCGs (Fig. 33. How galaxies are assembled and the role of gas dissipation affect the correlations (or lack thereof) among $M_{\mathrm{BH}}, \sigma$, and $L$. Simulations of mergers of gas-rich disk galaxies are able to produce remnant galaxies that follow the observed $M_{\mathrm{BH}}-\sigma$ correlation in Fig. 3a, over the intermediate mass range $M_{\mathrm{BH}} \approx 10^{7}-10^{9} M_{\odot}$ (refs 18, 19). By contrast, simulated mergers of elliptical galaxies with low-angular momentum progenitor orbits increase $M_{\mathrm{BH}}$ and $L$ by similar numerical factors, without increasing the velocity dispersion 20. Because these mergers are a likely path to forming the most massive galaxies, the $M_{\mathrm{BH}}-\sigma$ correlation may steepen or disappear altogether at the highest galaxy masses. Massive elliptical galaxies retain residual quantities of gas even after the decline of star formation. Accretion of this gas onto the galaxies' central black holes could help increase $M_{\mathrm{BH}}$ and further steepen the $M_{\mathrm{BH}}-\sigma$ and $M_{\mathrm{BH}}-L$ relations.

Black holes in excess of $10^{10} M_{\odot}$ are observed as quasars in the early Universe, from $1.4 \times 10^{9}$ to $3.3 \times 10^{9} \mathrm{yr}$ after the Big Bang ${ }^{[2}$ (redshift, $z=2-4.5$ ). Throughout the last $1.0 \times 10^{10} \mathrm{yr}$, however, these extremely massive black holes have not been accreting appreciably, and the average mass of the black holes powering quasars has decreased steadily. Quasar activity and elliptical galaxy formation are predicted to arise from similar merger-triggered processes, and there is growing evidence that present-day massive elliptical galaxies once hosted the most luminous high-redshift quasars 21 . Yet definitive classification of these quasars' host galaxies has remained elusive.

Our measurements of black holes with masses of around $10^{10} M_{\odot}$ in NGC 3842 and NGC 4889 provide circumstantial evidence that BCGs host the remnants of extremely luminous quasars. The number density of nearby BCGs $\left(\sim 5 \times 10^{-6} \mathrm{Mpc}^{-3}\right)$ is consistent with the number density of black holes $\left(\sim 3 \times 10^{-7}\right.$ to $\left.10^{-5} \mathrm{Mpc}^{-3}\right)$ with masses between $10^{9} M_{\odot}$ and $10^{10} M_{\odot}$ predicted from the $M_{\mathrm{BH}}-L$ relation and the luminosity function of nearby galaxies. Furthermore, both quantities agree with predictions based on the black hole masses and duty cycles of quasars. The black hole number density predicted from the $M_{\mathrm{BH}}-\sigma$ relation, however, is an order of magnitude less than the inferred quasar population 14,222. These two predictions can be reconciled if the $M_{\mathrm{BH}}-\sigma$ relation has upward curvature or a large degree of intrinsic scatter in $M_{\mathrm{BH}}$ at the highmass end, as suggested by our new measurements. With improvements in adaptive optics instrumentation on large optical telescopes and very-long baseline interferometry at radio wavelengths, black holes are being sought and detected in increasingly exotic host galaxies. Along with our measurements of the black hole masses in NGC 3842 and NGC 4889, future measurements in other massive galaxies will quantify the cumulative growth of supermassive black holes in the Universe's densest environments.

\section{References}

1. Netzer, H. The largest black holes and the most luminous galaxies. Astrophys. J. 583, L5-L8 (2003).

2. Vestergaard, H., Fan, X., Tremonti, C. A., Osmer, P. S. \& Richards, G. T. Mass functions of the active black holes in distant quasars from the Sloan Digital Sky Survey data release 3. Astrophys. J. 674, L1-L4 (2008).

3. Sargent, W. L. W., et al. Dynamical evidence for a central mass concentration in the galaxy M87. Astrophys. J. 221, 731-744 (1978).

4. Gebhardt, K., et al. The black hole mass in M87 from Gemini/NIFS adaptive optics observations. Astrophys. J. 729, 119 (2011).

5. Dressler, A. Observational evidence for supermassive black holes. In D. E. Osterbrock, \& J. S. Miller (ed.) Dark Matter in Galaxies, vol. 134 of IAU Symposium, 217 (1989).

6. Kormendy, J. \& Richstone, D. Inward bound the search for supermassive black holes in galactic nuclei. Ann. Rev. Astron. Astrophys. 33, 581-624 (1995).

7. Ferrarese, L. \& Merritt, D. A fundamental relation between supermassive black holes and their host galaxies. Astrophys. J. 539, L9-L12 (2000).

8. Gebhardt, K., et al. A relationship between nuclear black hole mass and galaxy velocity dispersion. Astrophys. J. 539, L13-L16 (2000).

9. Gültekin, K., et al. The $M_{\mathrm{BH}}-\sigma$ and $M_{\mathrm{BH}}-L$ relations in galactic bulges, and determinations of their intrinsic scatter. Astrophys. J. 698, 198-221 (2009).

10. Laine, S., et al. Hubble Space Telescope imaging of brightest cluster galaxies. Astron. J. 125, 478-505 (2003).

11. Postman, M. \& Lauer, T. R. Brightest cluster galaxies as standard candles. Astrophys. J. 440, 28-47 (1995).

12. Schwarzschild, M. A numerical model for a triaxial stellar system in dynamical equilibrium. Astrophys. J. 232, 236-247 (1979).

13. Loubser, S. I., Sansom, A. E., Sánchez-Blázquez, P., Soechting, I. K. \& Bromage, G. E., Radial kinematics of brightest cluster galaxies. Mon. Not. R. Astron. Soc. 391, 1009-1028 (2008).

14. Lauer, T. R., et al. The masses of nuclear black holes in luminous elliptical galaxies and implications for the space density of the most massive black holes. Astrophys. J. 662, 808-834 (2007).

15. Dalla Bontà, E., et al. The high-mass end of the black hole mass function: mass estimates in brightest cluster galaxies. Astrophys. J. 690, 537-559 (2009).

16. McConnell, N. J., et al. The The black hole mass in brightest cluster galaxy NGC 6086. Astrophys. J. 728, 100 (2011).

17. Nowak, N., et al. The supermassive black hole of Fornax A. Mon. Not. R. Astron. Soc. 391, 1629-1649 (2008).

18. Di Matteo, T., Volker, S., \& Hernquist, L. Energy input from quasars regulates the growth and activity of black holes and their host galaxies Nature 433, 604-607 (2005).

19. Robertson, B., et al. The Evolution of the $M_{\mathrm{BH}}-\sigma$ Relation. Astrophys. J. 641, 90-102 (2006)

20. Boylan-Kolchin, M., Ma, C.-P., \& Quataert, E. Red mergers and the assembly of massive elliptical galaxies: the fundamental plane and its projections. Mon. Not. R. Astron. Soc. 369, 1081-1089 (2006).

21. Hopkins, P. F., Bundy, K., Hernquist, L. \& Ellis, R. S. Observational evidence for the coevolution of galaxy mergers, quasars, and the blue/red galaxy transition. Astrophys. J. 659, 976-996 (2007).

22. Lauer, T. R., Tremaine, S., Richstone, D., \& Faber, S. M. Selection bias in observing the cosmological evolution of the $M_{\mathrm{BH}}-\sigma$ and $M_{\mathrm{BH}}-L$ relationships. Astrophys. J. 670, 249-260 (2007)

23. Allington-Smith, J., et al. Integral field spectroscopy with the Gemini Multi-Object Spectrograph. I. Design, construction, and testing. Pub. Astron. Soc. Pacific 115, 892-912 (2002)

24. Hill, G. J., et al. Design, construction, and performance of VIRUS-P: the prototype of a highly replicated integral field spectrograph for the HET. Proc. Soc. Photooptical Instrumentation Engineers 7014, 701470 (2008).

25. Larkin, J., et al. OSIRIS: a diffraction limited integral field spectrograph for Keck. Proc. Soc. Photo-optical Instrumentation Engineers 6269, 62691A (2006).

Supplementary Information http://www.nature.com/nature is linked to the online version of the paper at 
Acknowledgments N.J.M., C.-P.M, K.G. and J.R.G. are supported by the National Science Foundation. C.-P.M. is supported by NASA and by the Miller Institute for Basic Research in Science, University of California, Berkeley. S.A.W. is supported by NASA through the Hubble Fellowship. Data presented here were obtained using Gemini Observatory, W.M. Keck Observatory, and McDonald Observatory. The Gemini Observatory is operated by the Association of Universities for Research in Astronomy, Inc., under a cooperative agreement with the National Science Foundation on behalf of the Gemini partnership. The W. M. Keck Observatory is operated as a scientific partnership among the California Institute of Technology, the University of California, and NASA. The McDonald Observatory is operated by the University of Texas at Austin. The instrument VIRUS-P was funded by G. and C. Mitchell. Stellar orbit models were run using the facilities at the Texas Advanced Computing Center at The University of Texas at Austin.

Author Contributions N.J.M. carried out the data analysis and modeling. N.J.M, C.-P.M. and S.A.W. wrote the manuscript. C.-P.M. compiled the data for Figure 3 and oversaw communication among co-authors. S.A.W. analyzed OSIRIS data of NGC 3842. K.G. provided GMOS data of NGC 3842 and NGC 4889. K.G. and D.O.R. developed the stellar orbit modeling code. J.D.M. provided VIRUS-P data of NGC 3842. T.R.L. provided photometric data and image analysis of NGC 3842 and NGC 4889. J.R.G. led the OSIRIS observing campaign for NGC 3842. All authors contributed to the interpretive analysis of the observations and the writing of the paper.

Competing Interests The authors declare that they have no competing financial interests.

Correspondence Correspondence and requests for materials should be addressed to N. McConnell (email: nmcc@berkeley.edu) or C.-P. Ma (cpma@berkeley.edu).
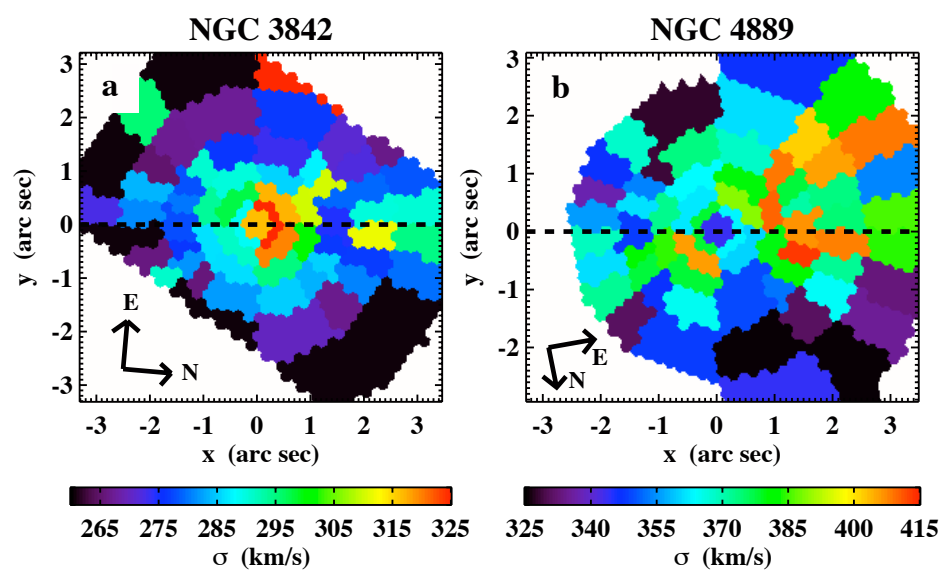

Figure 1 | Two-dimensional maps of the line-of-sight stellar velocity dispersions. The maps show the central regions of NGC 3842 (a) and NGC 4889 (b) observed using the GMOS spectrograph ${ }^{23}$ on the 8-m Gemini North telescope. Additional kinematics at large radii were measured using the VIRUS-P spectrograph $^{24}$ at the 2.7-m Harlan J. Smith telescope, and additional high-resolution data were acquired with the OSIRIS spectrograph ${ }^{25}$ at the $10-\mathrm{m}$ Keck 2 telescope. GMOS, OSIRIS, and VIRUS-P are all integral field spectrographs, which record spectra at multiple positions in a two-dimensional spatial array. The horizontal dashed line in each panel traces the major axis of the galaxy. The median 1 s.d. errors in velocity dispersion are $12 \mathrm{~km} \mathrm{~s}^{-1}$ and $20 \mathrm{~km} \mathrm{~s}^{-1}$ for NGC 3842 and NGC 4889, respectively. In NGC 4889 the highest velocity dispersions, near $410 \mathrm{~km} \mathrm{~s}^{-1}$, are located on the east side of the galaxy, at least 1.1 arcsec from the centre.
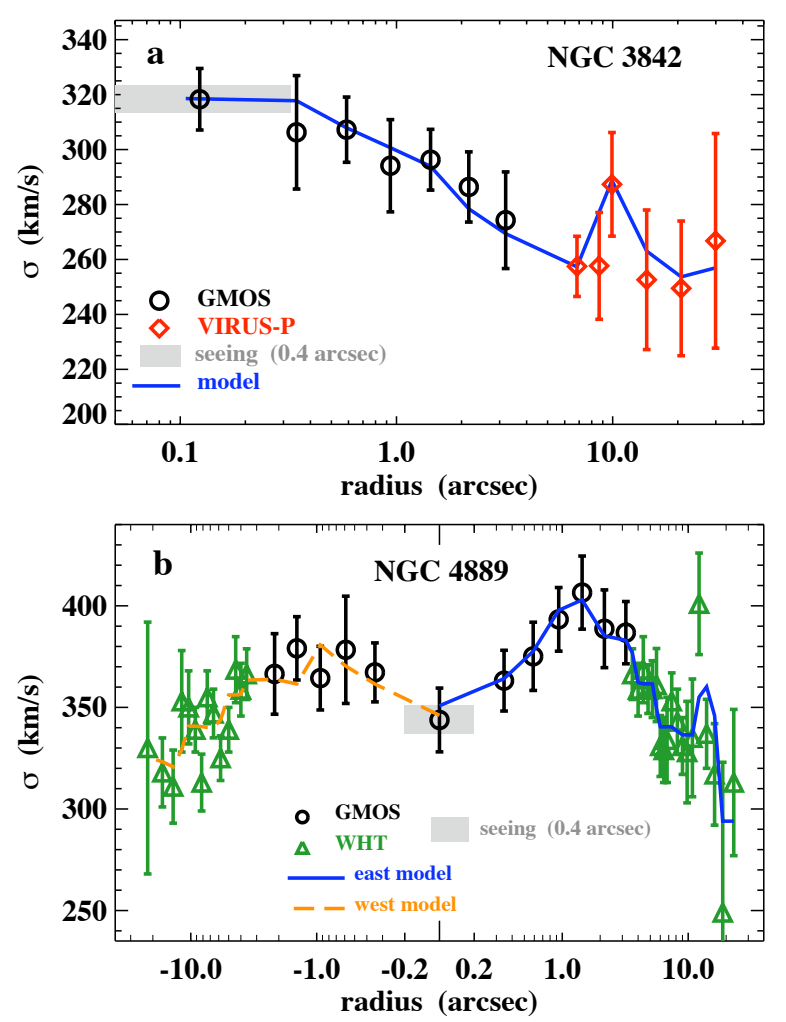

Figure 2 | One-dimensional profiles of the line-of-sight velocity dispersions. (a) Dispersion versus radius in NGC 3842, after averaging data at a given radius, based on measurements with GMOS (black circles) and VIRUS-P (red diamonds). The solid blue line is the projected line-of-sight dispersion from our best-fitting stellar orbit model of NGC 3842. (b) Dispersion versus radius along the major axis of NGC 4889, measured from GMOS (black circles) and the William Herschel Telescope ${ }^{[13]}$ (green diamonds). The maximum velocity dispersion occurs at $r=1.4$ arcsec. The solid blue line is the projected lineof-sight dispersion from our best-fitting orbit model using data from the east side of NGC $4889(r>0)$. The dashed orange line is from our best-fitting orbit model using data from the west side of NGC $4889(r<0)$. Error bars, 1 s.d. 

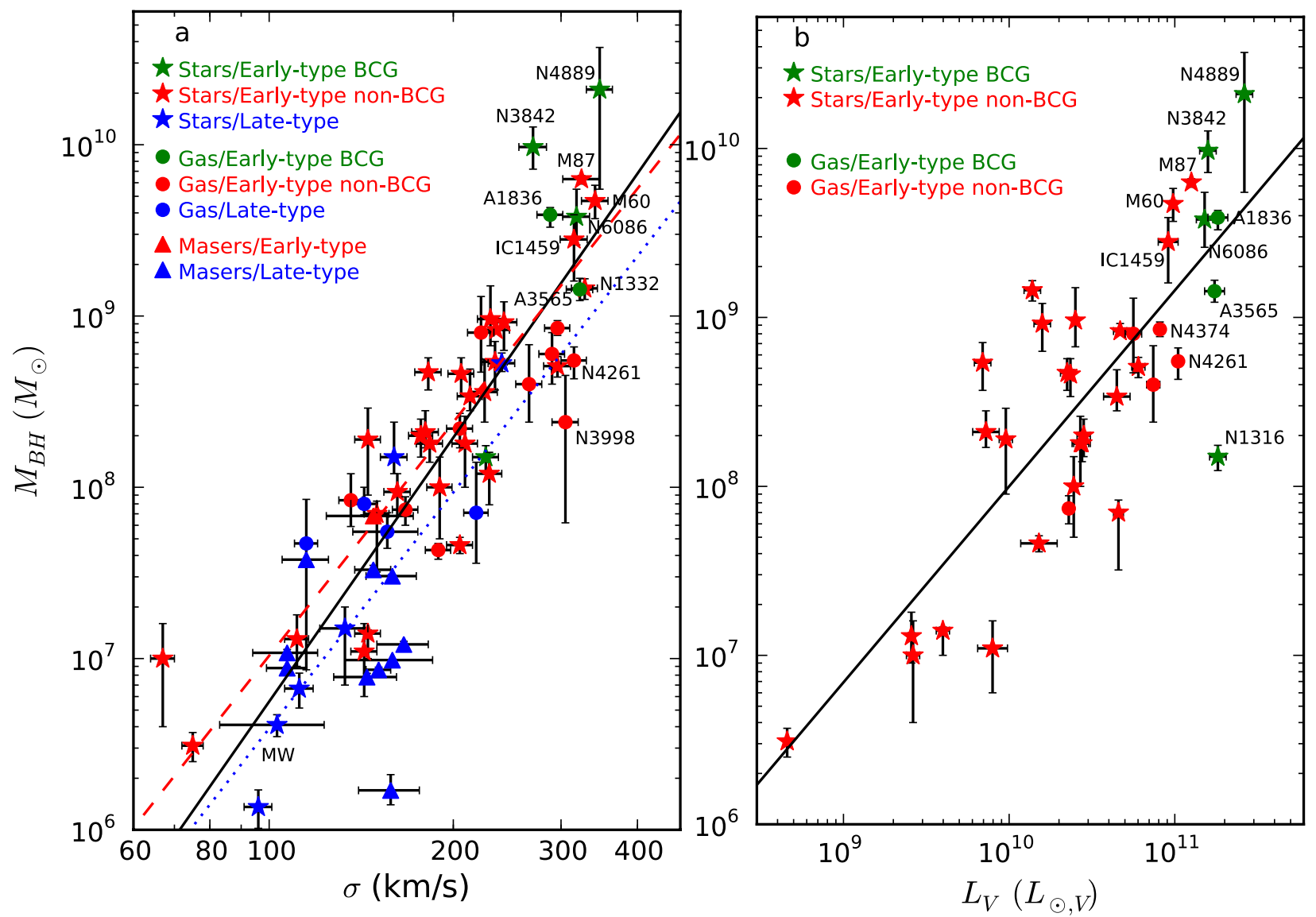

Figure 3 | Correlations of dynamically measured black hole masses and bulk properties of host galaxies. (a) Black hole mass, $M_{\mathrm{BH}}$, versus stellar velocity dispersion, $\sigma$, for 65 galaxies with direct dynamical measurements of $M_{\mathrm{BH}}$. For galaxies with spatially resolved stellar kinematics, $\sigma$ is the luminosity-weighted average within one effective radius (Supplementary Information). (b) Black hole mass versus $V$-band bulge luminosity, $L_{V}$ ( $L_{\odot, V}$, solar value), for 36 earlytype galaxies with direct dynamical measurements of $M_{\mathrm{BH}}$. Our sample of 65 galaxies consists of 32 measurements from a 2009 compilation 9 , 16 galaxies with masses updated since 2009, 15 new galaxies with $M_{\mathrm{BH}}$ measurements, and the two galaxies reported here. A complete list of the galaxies is given in Supplementary Table 4. BCGs (defined here as the most luminous galaxy in a cluster) are plotted in green, other elliptical and S0 galaxies are plotted in red, and late-type spiral galaxies are plotted in blue. The black hole masses are measured using dynamics of masers (triangles), stars (stars), or gas (circles). Error bars, $68 \%$ confidence intervals. For most of the maser galaxies, the error bars in $M_{\mathrm{BH}}$ are smaller than the plotted symbol. The solid black line in (a) shows the best-fitting power law for the entire sample: $\log _{10}\left(M_{\mathrm{BH}} / M_{\odot}\right)=8.29+5.12 \log _{10}\left[\sigma /\left(200 \mathrm{~km} \mathrm{~s}^{-1}\right)\right]$. When early-type and late-type galaxies are fit separately, the resulting power laws are $\log _{10}\left(M_{\mathrm{BH}} / M_{\odot}\right)=8.38+4.53 \log _{10}\left[\sigma /\left(200 \mathrm{~km} \mathrm{~s}^{-1}\right)\right]$ for elliptical and S0 galaxies (dashed red line), and $\log _{10}\left(M_{\mathrm{BH}} / M_{\odot}\right)=7.97+4.58 \log _{10}\left[\sigma /\left(200 \mathrm{~km} \mathrm{~s}^{-1}\right)\right]$ for spiral galaxies (dotted blue line). The solid black line in (b) shows the best-fitting power law: $\log _{10}\left(M_{\mathrm{BH}} / M_{\odot}\right)=9.16+1.16 \log _{10}\left(L_{V} / 10^{11} L_{\odot}\right)$. We do not label Messier 87 as a BCG, as is commonly done, as NGC 4472 in the Virgo cluster is 0.2 mag brighter. 


\section{Supplementary Information}

In the first section, we describe spectroscopic data of NGC 3842 and NGC 4889 and our procedures for measuring stellar kinematics. In the second section, we describe photometric data. In the third section we compare overlapping kinematic measurements from GMOS, OSIRIS, VIRUS$\mathrm{P}$, and long-slit spectroscopy. The fourth section contains a summary of our stellar orbit models. In the fifth section we describe the unusual kinematics and stellar orbital structures in NGC 4889, and justify our measurement of $M_{\mathrm{BH}}$. In the final section we describe our updated sample and fits to the $M_{\mathrm{BH}}-\sigma$ and $M_{\mathrm{BH}}-L$ relations.

We tabulate our spectroscopic observations, our results from modeling each quadrant of NGC 4889 , our fits to the $M_{\mathrm{BH}}-\sigma$ and $M_{\mathrm{BH}}-L$ relations, and the galaxy sample used to fit the relations. Our measured lineof-sight velocity distributions (LOSVDs) for NGC 3842 and NGC 4889 are available as online data.

\section{Spectroscopic Data and LOSVD Extraction}

We map stellar orbital motions in NGC 3842 and NGC 4889 by measuring the LOSVD for different regions in each galaxy. Each LOSVD is determined by fitting a composite template stellar spectrum to a fully reduced spectrum of the galaxy. The LOSVDs are non-parametric probability distributions, defined at each of 15 velocity bins. We use a Maximum Penalized Likelihood technique to optimize the LOSVD value in each velocity bin while simultaneously optimizing the weights of individual template stars $17,|26| 27$.

In Table 1 we summarize our observations with the integral field spectrographs GMOS, OSIRIS, and VIRUS-P. Figures 4,6 illustrate a sample galaxy and template spectrum from each instrument. Our data from GMOS only cover radii within 3.8 arcseconds ( $1.8 \mathrm{kpc}$ ) of the center of NGC 3842 and NGC 4889, and by themselves cannot fully remove the degeneracies between $M_{\mathrm{BH}}$ and $M_{\star} / L$. For NGC 3842, our VIRUS-P measurements cover radii out to 35.3 arcseconds $(16.8 \mathrm{kpc})$ and can distinguish the enclosed stellar mass profile from the galaxy's dark matter halo. This allows for an accurate determination of $M_{\star} / L$, such that the GMOS data can accurately constrain $M_{\mathrm{BH}}$. At radii from 3.6 to 23.0 arcseconds $(1.8-11.5$ kpc) along the major axis of NGC 4889, we use Gaussian velocity profiles from Loubser et al. (2008) ${ }^{13}$.

Our GMOS spectra for NGC 3842 and NGC 4889 are centered on the calcium triplet absorption lines near $860 \mathrm{~nm}$. A sample GMOS spectrum for each galaxy is shown in Figure 4 demonstrating the clean line profiles that are typical for this spectral region. Another advantage to using the calcium triplet is that kinematic measurements are not highly sensitive to the stellar template used 28 .

OSIRIS data of NGC 3842 were acquired with the 0.05 arcsecond spatial scale and the broad $H$-band filter, which spans a large number of atomic and molecular absorption features. To measure kinematics, we fit carbon monoxide band heads at 1598, 1619, 1640, and $1661 \mathrm{~nm}$, and a deep magnesium feature near $1500 \mathrm{~nm}$. The most severe source of noise in our OSIRIS spectra is residual narrow-line emission from the night sky. This background emission varies rapidly and is only partially corrected by recording 15-minute sky frames in between pairs of 15-minute science exposures. Even when masked, the contaminated channels represent a nonnegligible loss of spectral information. This loss must be countered by increasing the signal-to-noise ratio in the usable parts of the spectrum. To achieve adequate signal-to-noise, we bin the data to the same spatial regions as the overlapping LOSVDs from GMOS. Each of the final bins contains approximately 80 OSIRIS spatial pixels.

VIRUS-P data were acquired in low-resolution mode, which provides broad wavelength coverage. The poorest instrumental resolution over our field is $0.56 \mathrm{~nm}$ full width at half-maximum (FWHM), which is adequate considering that stellar velocity dispersions in NGC 3842 are typically near $250 \mathrm{~km} \mathrm{~s}^{-1}$, corresponding to a resolution of approximately $0.7 \mathrm{~nm}$ FWHM $\times(\lambda / 360 \mathrm{~nm})$. We fit VIRUS-P spectra with 16 template stars from the Indo-US library ${ }^{29}$, spanning spectral types from B9 to M3 and including stars with sub-solar and super-solar metallicities. However, some spectral regions require additional adjustments to account for metallicities and elemental abundance ratios outside the range of our template library. We do not attempt to fit the entire spectral range simultaneously, but instead follow the same fitting procedure used by Murphy et al. (2011) ${ }^{\frac{30}{3}}$ for VIRUS-P data of M87. Each spectrum is divided into four sub-regions, as illustrated in Figure 6 We independently determine the best-fit LOSVD for each sub-region and discard any sub-regions that fail to produce a believable fit. We then average the LOSVDs derived from individual spectral sub-regions.

Our stellar orbit models are axisymmetric, and so each of our final LOSVDs must represent an average over four quadrants of the galaxy. In order to preserve any rotational signal along the major axis, we invert the velocities from LOSVDs on the south side of NGC 3842 and the west side of NGC 4889. However, neither galaxy shows strong rotation. In

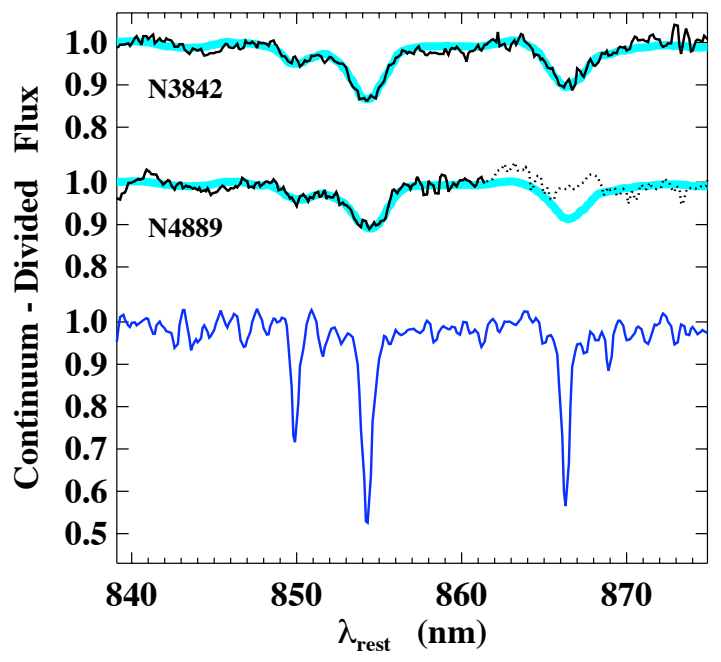

Figure 4 | GMOS spectra of NGC 3842 and NGC 4889. Each spectrum corresponds to the center of the galaxy ( $r<0.25$ arcseconds). The upper spectrum is NGC 3842 (black), overlaid with the best-fitting, LOSVD-convolved template spectrum (thick, light blue). The middle spectrum is NGC 4889, overlaid with the best-fitting template spectrum. The dotted portion of the spectrum was excluded from the LOSVD fitting. The lower spectrum is template star HD 73710 (G9III), before convolution with the LOSVD.

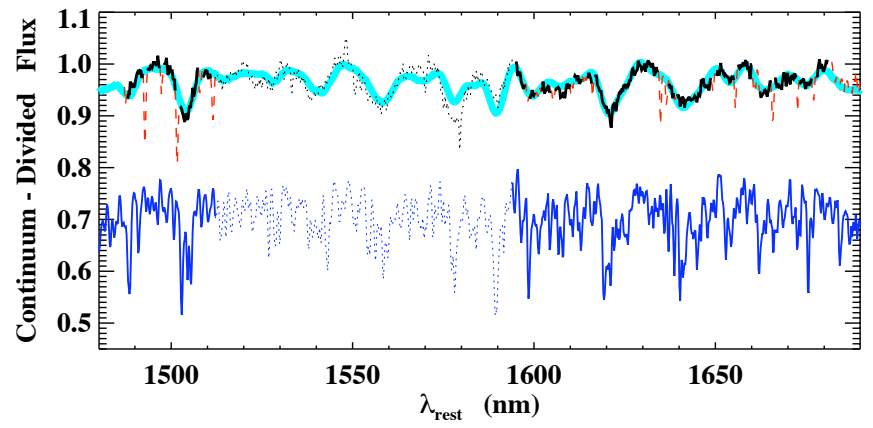

Figure 5 | OSIRIS spectrum of the central region of NGC $3842(r<0.25$ arcseconds, or $110 \mathrm{pc}$ ). The upper spectrum is NGC 3842 (black), overlaid with the best-fitting, LOSVD-convolved template spectrum (thick, light blue). The red dashed lines in the galaxy spectrum are residuals from imperfectly subtracted sky emission. The lower spectrum is the best-fit composite template before convolution with the LOSVD. Our observed template stars fit spectra of NGC 3842 poorly across the dotted region from 1510 to $1590 \mathrm{~nm}$, and therefore this region is excluded from kinematic fitting. 
NGC 3842, the resulting kinematics are sufficiently symmetric to average the LOSVDs from opposite sides of the galaxy. In NGC 4889, we have modeled four quadrants of the galaxy independently.

Our stellar orbit models require an estimate of the point spread function (PSF) for each instrument at the time of spectroscopic observations. We estimate the PSF for our GMOS data for both NGC 3842 and NGC 4889 from wide-field images taken during target acquisition. Although the models discussed herein assume a 0.4-arcsecond PSF for GMOS data, we have run a small number of models with a 0.7 -arcseond PSF and have found no significant changes in our results. While observing NGC 3842, we switched from the OSIRIS spectrograph to the OSIRIS imaging camera every few hours, and observed the adaptive optics tip/tilt star. We can tolerate a large degree of uncertainty in our measured PSF, as we have rebinned OSIRIS data of NGC 3842 to spatial scales that are several times coarser than the diffraction-limited FWHM.

\section{Photometric Data}

Our stellar orbit models are constrained to reproduce the observed stellar light profile of each galaxy, which requires accurate measurements of each galaxy's surface brightness profile over a large radial range. For radii out to 10 arcseconds, we adopt high-resolution $I$-band $(800 \mathrm{~nm})$ surface brightness profiles, obtained with WFPC 2 on the Hubble Space Telescope, and deconvolved with the instrumental PSF $\stackrel{10}{ }$. At larger radii out to 115 arcseconds, we use $R$-band $(600 \mathrm{~nm})$ data obtained with the $2.1 \mathrm{~m}$ telescope at Kitt Peak National Observatory (KPNO). The KPNO data have a field of view of $5.2 \times 5.2$ arcminutes, which enables accurate sky subtraction. We combine the individual profiles from WFPC2 and KPNO data at overlapping radii between 5 and 10 arcseconds, accounting for the average $R-I$ color over these radii. To compute the luminosity density profile of each galaxy, we deproject the surface brightness profile while assuming spheroidal isodensity contours 31 .
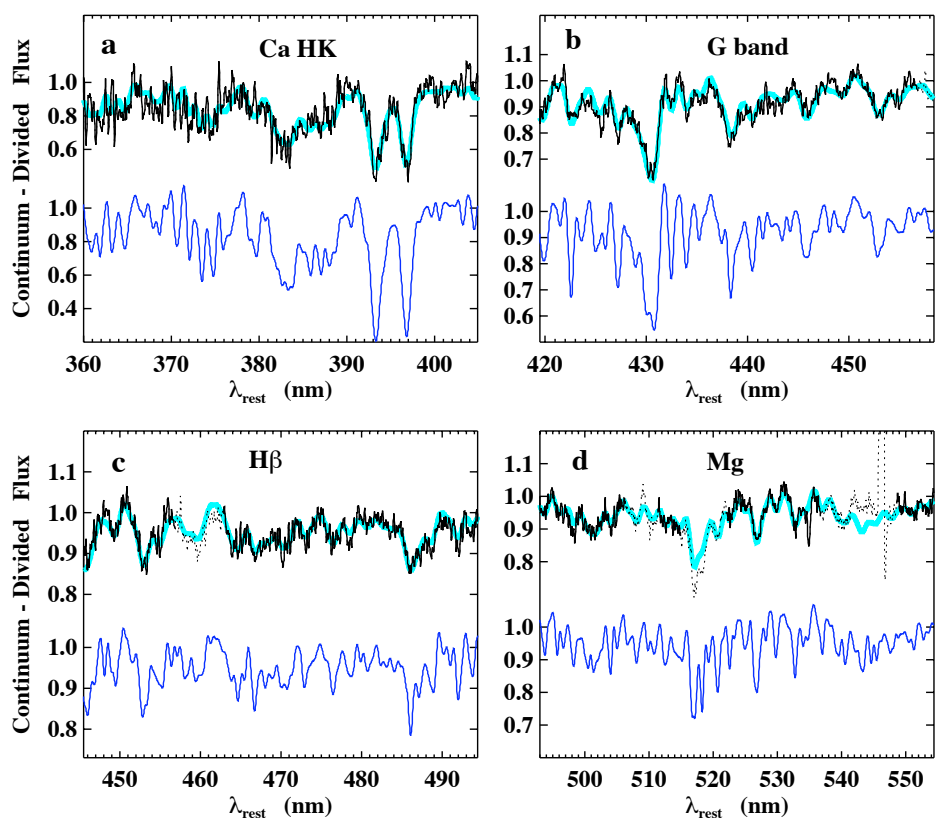

Figure 6 | VIRUS-P spectrum of NGC 3842, corresponding to a semi-annulus with an inner radius of 17.0 arcseconds $(7.7 \mathrm{kpc})$ and an outer radius of 24.5 arcseconds $(11.0 \mathrm{kpc})$. The four panels contain different sub-regions of the galaxy spectrum. Each sub-region is evaluated independently for a bestfit LOSVD and best-fit composite template spectrum. The upper spectrum in each panel is NGC 3842 (black), overlaid with the best-fitting, LOSVDconvolved template spectrum (thick, light blue). Dotted portions of the galaxy spectrum have been masked from the fit. The lower spectrum in each panel is the best-fit composite template before convolution with the LOSVD.

\section{Kinematic Data from Different Instruments}

Stellar kinematics along the major axes of NGC 3842 and NGC 4889 were previously measured by Loubser et al. (2008) 13 , using the ISIS longslit spectrograph on the William Herschel Telescope (WHT). The WHT measurements of NGC 3842 are in good agreement with our GMOS measurements for $r \leq 3.0$ arcseconds. The WHT data for NGC 4889 agree with our GMOS measurements on the west side of NGC 4889 and at radii between 1 and 2.4 arcseconds on the east side. However, they do not reproduce our measurement of the large central drop in stellar velocity dispersion (Figure 7). For a more direct comparison, we have rebinned our integral-field spectra to match the 0.4-arcsecond spatial sampling and 1.0arcsecond slit width of the WHT data. We find that rebinning alleviates the velocity dispersion discrepancy for all but the central point $(r=0)$. Our detection of a significantly sharper decrease in velocity dispersion is consistent with the superior seeing conditions of our data $(0.4$ arcseconds for GMOS, versus 1.0 arcseconds for WHT). Overall, we find our kinematic measurements in NGC 3842 and NGC 4889 to be broadly consistent with the independent measurements by Loubser et al., indicating that our kinematic extraction method has low systematic errors.

For N3842, our VIRUS-P measurements are also consistent with WHT measurements, which extend along the major axis to $r=20.8$ arcseconds. We prefer using data from VIRUS-P in our stellar orbit models because they extend to larger radii and provide full two-dimensional spatial sampling.

Near the center of NGC 3842, OSIRIS and GMOS provide independent measurements of stellar kinematics. We have binned data from OSIRIS and GMOS at identical spatial scales out to $r=0.7$ arcseconds $(330 \mathrm{pc})$ and have run orbit models fitting LOSVDs from OSIRIS and

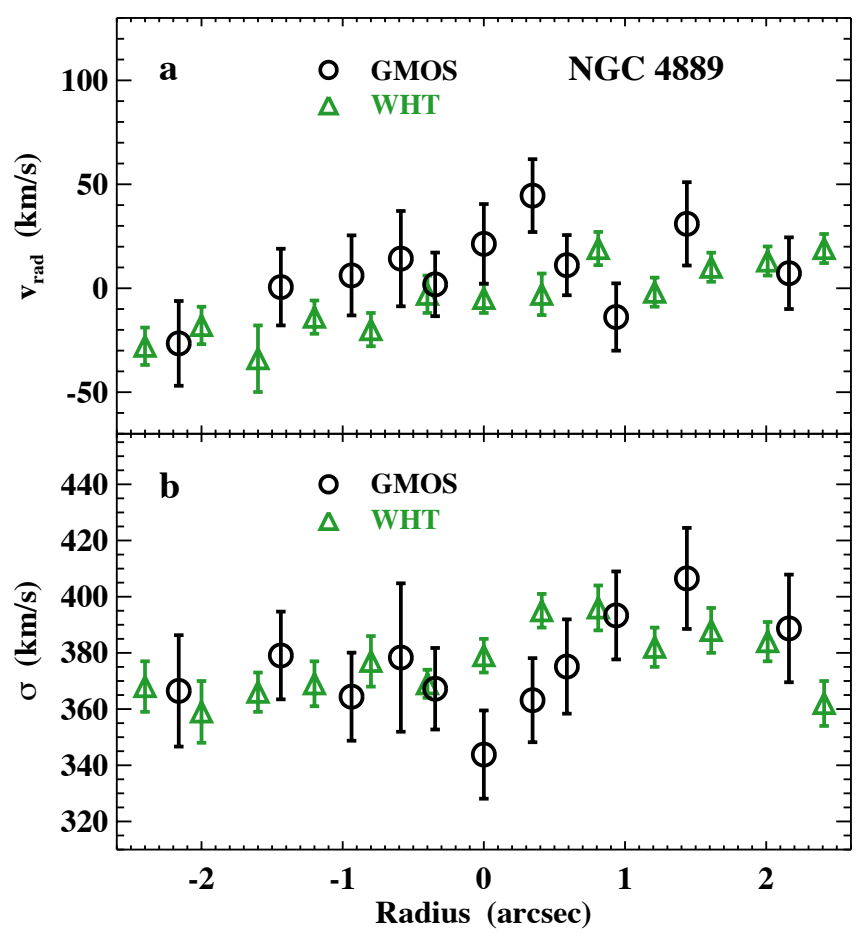

Figure 7 | (a) Radial velocity vs. radius in NGC 4889. (b) Velocity dispersion vs. radius. Green triangles are measurements from Loubser et al. (2008) 13 , using the ISIS spectrograph on the William Herschel Telescope (WHT). Black circles are our measurements using the GMOS intrgral-field unit. Error bars represent one standard deviation. On the west side and at radii from 1.0 to 2.4 arcseconds on the east side, the GMOS and WHT measurements agree within errors. At $r<1.0$ arcseconds on the east side, only GMOS detects a local minimum in velocity dispersion. The central feature might be unresolved in the WHT data due to worse seeing (1.0 arcseconds). 
GMOS simultaneously (as well as VIRUS-P data at large radii). Including the OSIRIS data causes the best-fit value of $M_{\mathrm{BH}}$ to decrease by up to $23 \%$, and the best-fit value of $M_{\star} / L$ to increase by as much as $8 \%$. This occurs because OSIRIS data show a less drastic increase in velocity dispersion than data from GMOS. In spite of these differences, results with and without OSIRIS data are consistent at the $68 \%$ confidence level.

Models fitting OSIRIS and GMOS data together yield higher average $\chi^{2}$ values per LOSVD. This is true even if we ignore the central regions where LOSVDs from OSIRIS and GMOS are not fully consistent. Even with several template stars, the overlapping absorption features in the H-band spectral region are difficult to model, and the LOSVDs derived from OSIRIS data may have systematic errors. Consequently, we judge the models with only GMOS and VIRUS-P data to be more reliable.

\section{Stellar Orbit Models and Statistical Analysis}

We generate models of NGC 3842 and NGC 4889 using Schwarzschild's method ${ }^{12}$, in which test particle orbits are computed in a static axisymmetric gravitational potential. We assume that each galaxy contains three mass components: stars, a central black hole, and an extended dark matter halo. The stellar mass density is assumed to follow the same profile as the observed luminosity density, with a constant stellar mass-to-light ratio, $M_{\star} / L$. Our modeling procedures are similar to those for NGC 6086, described in McConnell et al. (2011) 16 .

Each orbit in the model is assigned a scalar weight, and the set of bestfit orbital weights is determined by comparing projected LOSVDs from the orbits to the observed LOSVDs for the galaxy. Each observed LOSVD spatially maps to a linear combination of bins within the model, according to the spatial boundaries of the corresponding spectrum and the PSF of the observations. A corresponding model LOSVD is computed from the projected velocity distributions of individual orbits in each spatial bin, the appropriate combination of spatial bins, and the orbital weights. The bestfit weights are determined by the method of maximum entropy ${ }^{32}$, with the fixed constraint that the summed spatial distribution of all weighted orbits must match the observed luminosity density profile. The essential output of each model is a measurement of $\chi^{2}$, which defines the goodness of fit between our observed LOSVDs and the model LOSVDs, using the optimal combination of orbital weights. We determine the best-fit values and confidence intervals in $M_{\mathrm{BH}}$ and $M_{\star} / L$ by evaluating the relative likelihood between models with different assumed values of $M_{\mathrm{BH}}$ and $M_{\star} / L$. Figure 8 illustrates the behavior of $\chi^{2}$ with respect to $M_{\mathrm{BH}}$ and $M_{\star} / L$, for models with our best fitting dark matter halo.

\section{Models of NGC 4889}

Stellar kinematics in NGC 4889 are asymmetric with respect to the major and minor axes of the galaxy. Integral-field data from GMOS reveals velocity dispersions above $410 \mathrm{~km} \mathrm{~s}^{-1}$ on the east side of the galaxy, while the velocity dispersion rarely exceeds $380 \mathrm{~km} \mathrm{~s}^{-1}$ on the west side. This asymmetry prevents NGC 4889 from being fully described by a single set of axisymmetric orbit models. In order to place upper and lower bounds on the central black hole mass, we have run four suites of models, each fitting kinematics from one projected quadrant of NGC 4889. The northeast, southeast, and northwest quadrants yield consistent black hole masses, spanning a $68 \%$ confidence interval of $M_{\mathrm{BH}}=1.0-3.7 \times 10^{10} M_{\odot}$. The southwest quadrant has a maximum velocity dispersion of $373 \mathrm{~km} / \mathrm{s}$ and yields a $68 \%$ confidence interval of $M_{\mathrm{BH}}=0.6-1.7 \times 10^{10} M_{\odot}$.

We have run an additional set of models to approximate $M_{\mathrm{BH}}$ in the case of an off-center black hole. We apply a constant spatial offset of 1.4 arcseconds $(700 \mathrm{pc})$ to the kinematics on the east side of the galaxy, such that the highest velocity dispersion is aligned with the center of the model gravitational potential. These models cannot be fully trusted because the kinematic and photometric data are misaligned. Still, the resulting $68 \%$ confidence interval for $M_{\mathrm{BH}}$ falls entirely within the range bracketed by the models from different quadrants. Results from individual trials are listed in Table 2 Figure 9 illustrates $\chi^{2}$ versus $M_{\mathrm{BH}}$ for each series of models, after marginalizing over $M_{\star} / L$.

Although three-dimensional stellar velocities must increase in the vicinity of a black hole, a deficiency of radial orbits can produce a central minimum in the line-of-sight velocity dispersion, as we observe in NGC 4889. Indeed, our best-fitting models of NGC 4889 exhibit tangential bias at small radii, as shown in Figure 10 In contrast, models without a black hole reproduce the central drop in velocity dispersion with a nearly

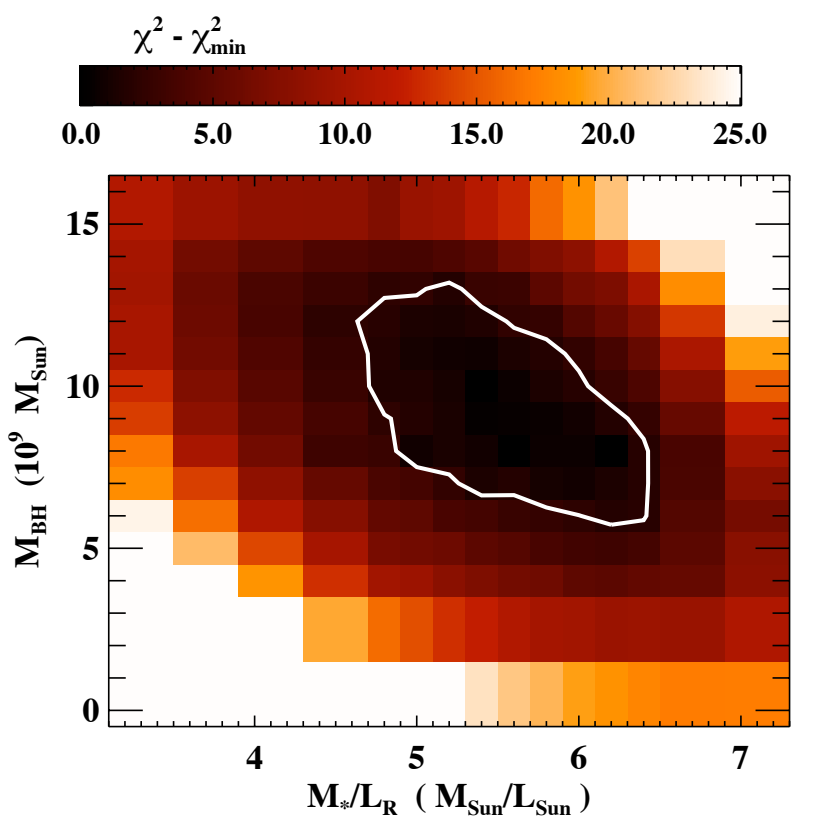

Figure 8 | Map of $\chi^{2}$ versus $M_{\mathrm{BH}}$ and $M_{\star} / L$ for stellar orbit models of NGC 3842. The models fit data from GMOS and VIRUS-P. The diagonal trend in $\chi^{2}$ indicates the degeneracy between stellar mass and black hole mass near the center of NGC 3842. For two free parameters with Gaussian likelihood distributions, the $68 \%$ confidence interval is defined where $\chi^{2}-\chi_{\min }^{2} \leq 2.30$, illustrated by the thick white contour. We obtain $68 \%$ confidence intervals of $7.2-12.7 \times 10^{9} M_{\odot}$ for $M_{\mathrm{BH}}$, and $4.4-5.8 M_{\odot} / L_{\odot}$ for $M_{\star} / L$. The median values, which we adopt as our final measurements, are $M_{\mathrm{BH}}=9.7 \times$ $10^{9} M_{\odot}$ and $M_{\star} / L=5.1 M_{\odot} / L_{\odot}$.

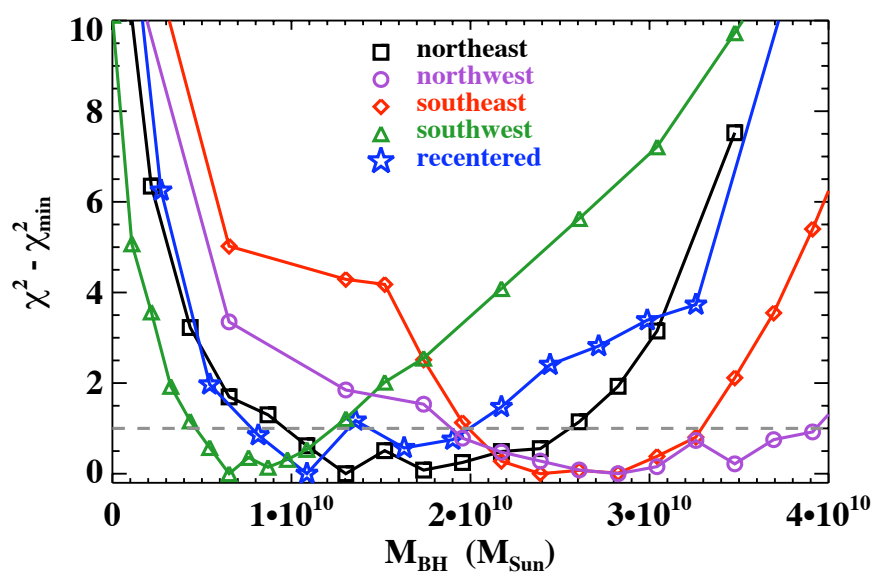

Figure $9 \mid \chi^{2}$ vs. $M_{\mathrm{BH}}$ for NGC 4889, after marginalizing over $M_{\star} / L$. Each line with symbols represents a models constrained by different set of LOSVDs from GMOS. Black squares, purple circles, red diamonds, and green triangles each use LOSVDs from a different quadrant of the galaxy. Blue stars represent models with spatially offset LOSVDs, to match the largest velocity dispersion with the center of the gravitational potential. Considering all models, the $68 \%$ confidence interval for $M_{\mathrm{BH}}$ is $0.6-3.7 \times 10^{10} M_{\odot}$. 
isotropic orbital distribution. However, these models yield a worse overall fit, indicated by higher values of $\chi^{2}$ in each quadrant. Models of NGC 3842 exhibit a similar but less severe trend, consistent with the modest increase in line-of-sight velocity dispersion toward the center.

The individual quadrants of NGC 4889 represent large variations in stellar kinematics, but each quadrant still partially constrains the enclosed mass within the central few arcseconds. By adopting the most extreme range of confidence limits, $M_{\mathrm{BH}}=0.6-3.7 \times 10^{10} M_{\odot}$, we only exclude black holes whose gravitational influence would contradict our entire field of data. Further extensions to this confidence interval should only reflect overall systematic biases. Large systematic biases in our kinematic measurements are unlikely, as demonstrated by their agreement with independent measurements by Loubser et al. (2008) 13 . Our models of NGC 4889 assume an edge-on inclination. This is indirectly supported by the observed axis ratio of 0.7 , which implies a relatively eccentric intrinsic shape even for an edge-on system. Models with a more face-on inclination might yield a systematically higher black hole mass 33 . A fundamental
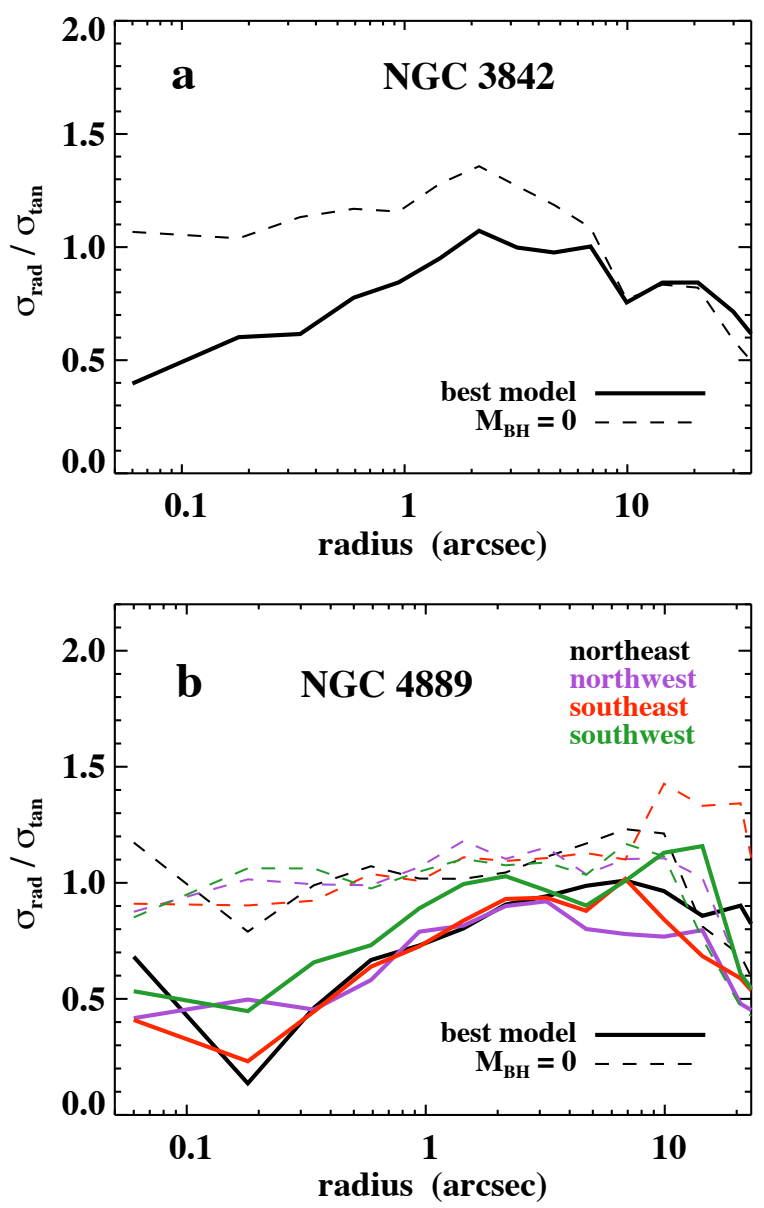

Figure 10 | Orbital anisotropy in models of NGC 3842 and NGC 4889. Threedimensional stellar velocities in the models are divided into radial and tangential components. The ratio of velocity dispersions, $\sigma_{\mathrm{rad}} / \sigma_{\mathrm{tan}}$, varies with radius and the assumed $M_{\mathrm{BH}}$ in the model. (a) The thick solid line represents the best-fitting model for NGC 3842, with $M_{\mathrm{BH}}=8.5 \times 10^{9} M_{\odot}$. The thin dashed line represents the best model with no black hole. (b) Thick solid lines represent the best-fitting model for each quadrant in NGC 4889, with $M_{\mathrm{BH}}=1.3 \times 10^{10} M_{\odot}$ (northeast), $2.8 \times 10^{10} M_{\odot}$ (northwest), $2.4 \times 10^{10} M_{\odot}$ (southeast), and $6.5 \times 10^{9} M_{\odot}$ (southwest). Thin dashed lines represent models with no black hole. For NGC 3842 and each quadrant of NGC 4889, our kinematic data are best fit with a massive black hole and a prevalence of tangential orbits at $r<2$ arcseconds (1 kpc). In NGC 4889, this tangential bias is responsible for the observed decrease in line-of-sight velocity dispersion near the center. assumption of all orbit superposition models is that the stellar motions reflect a steady-state gravitational potential, rather than transient conditions. These models could misrepresent the range of allowed black hole masses if the observed kinematics in NGC 4889 reflected a temporary phenomenon such as an ongoing galaxy merger. NGC 4889 appears photometrically undisturbed, reducing the likelihood of such an event.

Because our adopted confidence interval places large error bars on $M_{\mathrm{BH}}$ in NGC 4889, this galaxy has relatively little weight in our fits to the $M_{\mathrm{BH}}-\sigma$ and $M_{\mathrm{BH}}-L$ relationships. A systematic error in our measurement would produce a minimal bias in the best-fit relations. Likewise, our discussion of steepening and scatter at the high-mass ends of the correlations depends upon several objects and is not highly sensitive to the measurement in NGC 4889.

Power-Law Fits to the $M_{\mathrm{BH}}-\sigma$ and $M_{\mathrm{BH}}-L$ Relations We revisit the $M_{\mathrm{BH}}-\sigma$ and $M_{\mathrm{BH}}-L$ relations by updating the sample of 49 black holes from Gültekin et al. (2009; hereafter G09) 9 , which was compiled from earlier studies. Including NGC 3842 and NGC 4889, we add 17 galaxies with recently measured black hole masses to the sample. The new objects include two more BCGs 1617 , eight active galactic nuclei with high-precision maser-based measurements 34,35 , and two galaxies with pseudobulges 36 . We also include updated black hole masses for 16 other galaxies in the 2009 sample, based on stellar orbit models with dark matter halos and more thorough orbit libraries 4/33]37|38. In particular, the revised masses for M60 and M87 are twice as large as the earlier values.

Our updated sample uses the same selection criteria as G09; in particular, only direct dynamical measurements of $M_{\mathrm{BH}}$ are included. G09 estimated galaxy distances by assuming a Hubble parameter $H_{0}=70 \mathrm{~km} \mathrm{~s}^{-1}$ $\mathrm{Mpc}^{-1}$, and rescaled their sample of black hole masses accordingly $\left(M_{\mathrm{BH}} \propto D\right)$. We have followed this convention for NGC 3842, NGC 4889 , and the rest of our sample. Our fits to $M_{\mathrm{BH}}(\sigma)$ do not include upper limits. Updated models of one galaxy, NGC 2778, do not produce a significant black hole detection; after removing this object, our updated sample contains 65 black hole masses.

We define $\sigma$ in the same manner as the 2009 sample. Wherever possible, we use the luminosity-weighted effective velocity dispersion, measured using spatially resolved data out to one effective radius. G09 found no evidence of systematic bias between this definition of $\sigma$ and more ubiquitous single-aperture measurements. Nonetheless, several galaxies in G09 have measurements of $\sigma$ that include data at very small radii, within which the central black hole directly influences the stellar velocity dispersion. This is inappropriate for studies that wish to treat $M_{\mathrm{BH}}$ and $\sigma$ as fundamentally independent variables. We have therefore re-evaluated $\sigma$ in three galaxies with large black hole masses and available spatially resolved kinematics, by excluding data within the black hole's radius of influence. We use this same treatment to measure $\sigma$ for NGC 3842 and NGC 4889. For M87, we adopt the updated values of $M_{\mathrm{BH}}$ and $\sigma$ from Gebhardt et al. $(2011)^{4}$. Following G09, we assume all measurements of $\sigma$ have an uncertainty of at least $5 \%$.

To fit the $M_{\mathrm{BH}}-L$ relationship, we only consider early-type galaxies for which the stellar luminosity of the spheroidal component can be measured reliably. Including NGC 3842, we add 6 galaxies to the $M_{\mathrm{BH}}-L$ sample of G09. We exclude NGC 2778. We also exclude NGC 3607 and NGC 4564, for which the literature contains large discrepancies in the measured luminosity 9214 . Our final sample for fitting $M_{\mathrm{BH}}-L$ contains 36 black hole masses.

Our fits to $M_{\mathrm{BH}}-\sigma$ and $M_{\mathrm{BH}}-L$ assume a single-index power law as the functional form of both relations, following the convention most commonly used in prior studies. Specifically, we define the $M_{\mathrm{BH}}-\sigma$ relationship to be $\log _{10}\left(M_{\mathrm{BH}} / M_{\odot}\right)=\alpha+\beta \log _{10}\left(\sigma / 200 \mathrm{~km} \mathrm{~s}^{-1}\right)$, and the $M_{\mathrm{BH}}-L$ relationship to be $\log _{10}\left(M_{\mathrm{BH}} / M_{\odot}\right)=\alpha+$ $\beta \log _{10}\left(L_{V} / 10^{11} L_{\odot}\right)$. For each relationship, we follow the method of 
Tremaine et al. (2002) ${ }^{39}$ to fit for $\alpha$ and $\beta$. We minimize the quantity

$$
\chi^{2}=\sum_{i=1}^{N} \frac{\left(M_{\mathrm{BH}, i}-\alpha-\beta \sigma_{i}\right)^{2}}{\epsilon_{0}^{2}+\epsilon_{M, i}^{2}+\beta^{2} \epsilon_{\sigma, i}^{2}}
$$

where $\epsilon_{\sigma}$ is the measurement error in $\sigma, \epsilon_{M}$ is the measurement error in $M_{\mathrm{BH}}$, and $\epsilon_{0}$ is the intrinsic scatter in the $M_{\mathrm{BH}}-\sigma$ relation. We set $\epsilon_{0}$ such that $\chi^{2}$ per degree of freedom is unity after minimization. The $68 \%$ confidence intervals for $\alpha$ and $\beta$ correspond to the maximum range of $\alpha$ and $\beta$ for which $\chi^{2}-\chi_{\min }^{2} \leq 1$.

We list our best-fit values of $\alpha, \beta$, and $\epsilon_{0}$ for various sub-samples in Table 3 We list our full sample of galaxies in Table 4. Graham et al. (2011) ${ }^{\frac{40}{40}}$ recently compiled a sample of 64 galaxies with dynamical black hole measurements and found a power-law index of 5.13 for the $M_{\mathrm{BH}}-\sigma$ relation, very similar to the index we report herein. However, only 52 galaxies appear in their sample as well as ours. Of the 13 galaxies that appear only in our sample, seven use precise maser-based measurements of $M_{\mathrm{BH}}{ }^{34,35}$, and three are BCGs. Additionally, 11 galaxies have received updated measurements of $M_{\mathrm{BH}}$ since the compilation of Graham et al. (2011), using stellar orbit models with dark matter halos and larger orbit libraries $\frac{38}{\text {. }}$.

\section{References}

26. Gebhardt, K., et al. Axisymmetric, three-integral models of galaxies: a massive black hole in NGC 3379. Astron. J. 119, 1157-1171 (2000).

27. Pinkney, J., et al. Kinematics of 10 early-type galaxies from Hubble Space Telescope and ground-based spectroscopy. Astrophys. J. 596, 903-929 (2003).

28. Barth, A. J., Ho, L. C., \& Sargent, W. L. W. A study of the direct fitting method for measurement of galaxy velocity dispersions. Astron. J. 124, 2607-2614 (2002).

29. Valdes, F., Gupta, R., Rose, J. A., Singh, H. P., \& Bell, D. J. The Indo-US library of Coudé feed stellar spectra. Astrophys. J. Supp. 152, 251-259 (2004).

30. Murphy, J. D., Gebhardt, K., \& Adams, J. J., Galaxy kinematics with VIRUS-P: the dark matter halo of M87. Astrophys. J. 729, 129 (2011).

31. Gebhardt, K., et al. The centers of early-type galaxies with HST. III. Non-parametric recovery of stellar luminosity distribution. Astron. J. 112, 105-113 (1996).

32. Richstone, D. O., \& Tremaine, S. Maximum-entropy models of galaxies. Astrophys. J. 327, 82-88 (1988)

33. van den Bosch, R. C. E. \& de Zeeuw, P. T. Estimating black hole masses in triaxial galaxies. Mon. Not. R. Astron. Soc. 401, 1770-1780 (2010).

34. Greene, J. E., et al. Precise black hole masses from megamaser disks: black holebulge relations at low mass. Astrophys. J. 721, 26-45 (2010).

35. Kuo, C. Y., et al. The megamaser cosmology project. III. Accurate masses of seven supermassive black holes in active galaxies with circumnuclear megamaser disks. Astrophys. J. 727, 20 (2011).

36. Kormendy, J., Bender, R., \& Cornell, M. E. Supermassive black holes do not correlate with galaxy disks or pseudobulges. Nature 469, 74-76 (2011).

37. Shen, J. \& Gebhardt, K. The supermassive black hole and dark matter halo of NGC 4649 (M60). Astrophys. J. 711, 484-494 (2010).

38. Schulze, A. \& Gebhardt, K. Effect of a dark matter halo on the determination of black hole masses. Astrophys. J. 729, 21 (2011).

39. Tremaine, S., et al. The slope of the black hole mass versus velocity dispersion correlation. Astrophys. J. 574, 740-753 (2002).

40. Graham, A. W., Onken, C. A., Athanassoula, E., \& Combes, F. An expanded $M_{\mathrm{BH}}-\sigma$ diagram, and a new calibration of active galactic nuclei masses. Mon. Not. R. Astron. Soc. 412, 2211-2228 (2011).

41. Ghez, A M.., et al. Measuring distance and properties of the Milky Way's central supermassive black hole with stellar orbits. Astrophys. J. 689, 1044-1062 (2008).

42. Gillessen, S., et al. Monitoring stellar orbits around the massive black hole in the galactic center. Astrophys. J. 692, 1075-1109 (2009)

43. Greenhill, L. J., et al. A warped accretion disk and wide-angle outflow in the inner parsec of the Circinus galaxy. Astrophys. J. 590, 162-173 (2003)

44. Cappellari, M., et al. The counterrotating core and the black hole mass of IC 1459. Astrophys. J. 578, 787-805 (2002).

45. verolme, E. K., et al. A SAURON study of M32: measuring the intrinsic flattening and the central black hole mass. Mon. Not. R. Astron. Soc. 335, 517-525 (2002).

46. Bender, R., et al. HST STIS spectroscopy of the triple nucleus of M31: two nested disks in Keplerian rotation around a supermassive black hole. Astrophys. J. 631, 280-300 (2005)

47. Krajnovic, D., McDermid, R. M., Cappellari, M., \& Davies, R. L. Determination of masses of the central black holes in NGC 524 and 2549 using laser guide star adaptive optics. Mon. Not. R. Astron. Soc. 399, 1839-1857 (2009).
48. Bower, G. A., et al. Evidence of a supermassive black hole in the galaxy NGC 1023 from nuclear stellar dynamics. Astrophys. J. 550, 75-86 (2001).

49. Lodato, G. \& Bertin, G. Non-Keplerian rotation in the nucleus of NGC 1068: evidence for a massive accretion disk?. Astron. Astrophys. 398, 517-524 (2003).

50. Atkinson, J. W., et al. Supermassive black hole mass measurements for NGC 1300 and 2748 based on Hubble Space Telescope emission-line gas kinematics. Mon. Not. R. Astron. Soc. 359, 504-520 (2005).

51. Rusli, S. P., et al. The central black hole mass of the high- $\sigma$ but low-bulge-luminosity lenticular galaxy NGC 1332. Mon. Not. R. Astron. Soc. 410, 1223-1236 (2011).

52. Gebhardt, K., et al. The black hole mass and extreme orbital structure in NGC 1399. Astrophys. J. 671, 1321-1328 (2007).

53. Houghton, R. C. W., et al. The central kinematics of NGC 1399 measured with 14 pc resolution. Mon. Not. R. Astron. Soc. 367, 2-18 (2006).

54. Sarzi, M., et al. Supermassive black holes in bulges. Astrophys. J. 550, 65-74 (2001).

55. Devereux, N., Ford, H., Tsvetanov, Z., \& Jacoby, G. STIS spectroscopy of the central 10 parsecs of M81: evidence for a massive black hole. Astron. J. 125, 1226=1235 (2003).

56. Emsellem, E., Dejonghe, H., \& Bacon, R. Dynamical models of NGC 3115. Mon. Not. R. Astron. Soc. 303, 495-514 (1999).

57. Davies, R. I., et al. The star-forming torus and stellar dynamical black hole mass in the Seyfert 1 nucleus of NGC 3227. Astrophys. J. 646, 754-773 (2006)

58. Barth, A. J., et al. Evidence for a supermassive black hole in the S0 galaxy NGC 3245. Astrophys. J. 555, 685-708 (2001).

59. Nowak, N., et al. Do black hole masses scale with classical bulge luminosities only? The case of the two composite pseudo-bulge galaxies NGC 3368 and NGC 3489 Mon. Not. R. Astron. Soc. 403, 646-672 (2010).

60. Kondratko, P. T., , Greenhill, L. J., \& Moran, J. M. The parsec-scale accretion disk in NGC 3393. Astrophys. J. 678, 87-95 (2008).

61. Gültekin, K., et al. A quintet of black hole mass determinations. Astrophys. J. 695 1577-1590 (2009).

62. de Francesco, G., Capetti, A., \& Marconi, A. Measuring supermassive black holes with gas kinematics: the active S0 galaxy NGC 3998. Astron. Astrophys. 460, 439448 (2006).

63. Herrnstein, J. R., Moran, J. M., Greenhill, L. J., \& Trotter, A. S. The geometry of and mass accretion rate through the maser accretion disk in NGC 4258. Astrophys. J. 429, 719-738 (2005)

64. Ferrarese, L. \& Ford, H. C. Nuclear disks of gas and dust in early-type galaxies and the hunt for massive black holes: Hubble Space Telescope observations of NGC 6251. Astrophys. J. 515, 583-602 (1996).

65. Cretton, N. \& van den Bosch, F. C. Evidence for a massive black hole in the S0 galaxy NGC 4342. Astrophys. J. 514, 704-724 (1999).

66. Walsh, J. L., , Barth, A. J., \& Sarzi, M. The supermassive black hole in M84 revisited. Astrophys. J. 721, 762-776 (2010)

67. Nowak, N., et al. The supermassive black hole in NGC 4486A detected with SINFONI at the Very Large Telescope. Mon. Not. R. Astron. Soc. 379, 909-914 (2007).

68. de Francesco, G., Capetti, A., \& Marconi, A. Measuring supermassive black holes with gas kinematics. II. The LINERs IC 989, NGC 5077, and NGC 6500. Astron. Astrophys. 479, 355-363 (2008)

69. Silge, J. D., Gebhardt, K., Bergmann, M., \& Richstone, D. Gemini Near Infrared Spectrograph observations of the central supermassive black hole in Centaurus A. Astron. J. 130, 406-417 (2005).

70. Cappellari, M., et al. The mass of the black hole in Centaurus A from SINFONI AO-assisted integral-field observations of stellar kinematics. Mon. Not. R. Astron. Soc. 394, 660-674 (2009).

71. Ferrarese, L., Ford, H. C., \& Jaffe, W. Evidence for a massive black hole in the active galaxy NGC 4261 from Hubble Space Telescope images and spectra. Astrophys. J. 470, 444-459 (1999).

72. van der Marel, R. P., \& van den Bosch, F. C. Evidence for a $3 \times 10^{8} M_{\odot}$ black hole in NGC 7052 from Hubble Space Telescope observations of the nuclear gas disk. Astron. J. 116, 2220-2236 (1998)

73. Wold, M., Lacy, M., Käufl, H. U., \& Siebenmorgen, R. The nuclear regions of NGC 7582 from [Ne II] spectroscopy at $12.8 \mu \mathrm{m}$ - an estimate of the black hole mass. Astron. Astrophys. 460, 449-457 (2006)

74. Braatz, J. A., et al. The megamaser cosmology project. II. The angular-diameter distance to UGC 3789. Astrophys. J. 718, 657-665 (2010).

75. Graham, A. W., Colless, M. M., Busarello, G., Zaggia, S., \& Longo, G. Extended stellar kinematics of elliptical galaxies in the Fornax cluster. Astron. Astrophys. Supp. 133, 325-336 (1998) 
Table 1 | Spectroscopic observations

\begin{tabular}{cccccccccc}
\hline Galaxy & Instrument & UT Date & $N_{\exp }$ & $\begin{array}{c}t_{\exp } \\
(\mathrm{s})\end{array}$ & $\begin{array}{c}\lambda \\
(\mathrm{nm})\end{array}$ & $\begin{array}{c}\Delta \lambda \\
(\mathrm{nm})\end{array}$ & $\begin{array}{c}r_{\max } \\
(\operatorname{arcsec})\end{array}$ & $\begin{array}{c}\Delta r \\
(\operatorname{arcsec})\end{array}$ & $\begin{array}{c}\text { PSF FWHM } \\
(\operatorname{arc~sec})\end{array}$ \\
\hline NGC 3842 & GMOS & April 27, 2003 & 5 & 1200 & $755-949$ & 0.25 & 3.8 & 0.2 & 0.4 \\
NGC 3842 & OSIRIS & May 8-10, 2010 & 16 & 900 & $1473-1803$ & 0.72 & 0.7 & 0.05 & 0.08 \\
NGC 3842 & VIRUS-P & March 7-8, 2011 & 3 & 1200 & $358-589$ & 0.52 & 35.3 & 4.1 & 2.0 \\
NGC 4889 & GMOS & March 13, 2003 & 5 & 1200 & $755-949$ & 0.25 & 3.8 & 0.2 & 0.4 \\
\hline
\end{tabular}

Notes: $N_{\exp }$ is the number of science exposures recorded. $t_{\exp }$ is the integration time per exposure. $\Delta \lambda$ is the median instrumental resolution (FWHM). $r_{\max }$ is the maximum radius of usable data, with respect to the center of the galaxy. $\Delta r$ is the instrumental spatial scale, equal to the angular diameter of one lenslet or fiber.

Table 2 | Models of NGC 4889

\begin{tabular}{ccccccc}
\hline Quadrant & $\begin{array}{c}M_{\mathrm{BH}} \\
\left(M_{\odot}\right)\end{array}$ & $\begin{array}{c}M_{\mathrm{BH}, \min } \\
\left(M_{\odot}\right)\end{array}$ & $\begin{array}{c}M_{\mathrm{BH}, \max } \\
\left(M_{\odot}\right)\end{array}$ & $\begin{array}{c}M_{\star} / L \\
\left(M_{\odot} / L_{\odot}\right)\end{array}$ & $\begin{array}{c}M_{\star} / L, \min \\
\left(M_{\odot} / L_{\odot}\right)\end{array}$ & $\begin{array}{c}M_{\star} / L, \max \\
\left(M_{\odot} / L_{\odot}\right)\end{array}$ \\
\hline \multirow{2}{*}{$\begin{array}{c} \\
\text { northeast }\end{array}$} & $1.7 \times 10^{10}$ & $1.0 \times 10^{10}$ & $2.5 \times 10^{10}$ & 6.1 & 4.6 & 7.3 \\
southeast & $2.6 \times 10^{10}$ & $2.0 \times 10^{10}$ & $3.2 \times 10^{10}$ & 5.6 & 4.2 & 6.7 \\
northwest & $2.7 \times 10^{10}$ & $1.6 \times 10^{10}$ & $3.7 \times 10^{10}$ & 5.8 & 4.4 & 7.0 \\
southwest & $9.8 \times 10^{9}$ & $5.5 \times 10^{9}$ & $1.7 \times 10^{10}$ & 6.6 & 5.3 & 7.6 \\
& & & & & \\
east & $2.9 \times 10^{10}$ & $2.1 \times 10^{10}$ & $3.4 \times 10^{10}$ & 5.4 & 4.5 & 6.4 \\
west & $1.2 \times 10^{10}$ & $6.5 \times 10^{9}$ & $2.0 \times 10^{10}$ & 6.4 & 5.2 & 7.4 \\
recentered & $1.5 \times 10^{10}$ & $8.7 \times 10^{9}$ & $2.4 \times 10^{10}$ & 6.5 & 5.4 & 7.3 \\
\hline
\end{tabular}

Notes: The "east" and "west" trials used LOSVDs from spectra that were binned symmetrically over the north and south sides of the galaxy. The "recentered" trial added an artificial position offset to the LOSVDs, such that the maximum velocity dispersion was placed at the center of the gravitational potential. $M_{\mathrm{BH}, \min }, M_{\mathrm{BH}, \max }, M_{\star} / L,_{\min }$, and $M_{\star} / L,_{\max }$ represent $68 \%$ confidence limits. $M_{\star} / L$ corresponds to $R$-band photometry.

Table 3 | Fits to $M_{\mathrm{BH}}-\sigma$ and $M_{\mathrm{BH}}-L$

\begin{tabular}{cccccc}
\hline Relationship & Sample & $N_{\text {gal }}$ & $\alpha$ & $\beta$ & $\epsilon_{0}$ \\
\hline & & & & & \\
$M_{\mathrm{BH}}-\sigma$ & all & 65 & $8.29 \pm 0.06$ & $5.12 \pm 0.36$ & 0.43 \\
$M_{\mathrm{BH}}-\sigma$ & early-type & 45 & $8.38 \pm 0.06$ & $4.53 \pm 0.40$ & 0.38 \\
$M_{\mathrm{BH}}-\sigma$ & late-type & 20 & $7.97 \pm 0.22$ & $4.58 \pm 1.25$ & 0.44 \\
$M_{\mathrm{BH}}-\sigma$ & ML & 36 & $8.43 \pm 0.07$ & $4.66 \pm 0.43$ & 0.38 \\
& & & & & \\
$M_{\mathrm{BH}}-L$ & $\mathrm{ML}$ & 36 & $9.16 \pm 0.11$ & $1.16 \pm 0.14$ & 0.50 \\
\hline
\end{tabular}

Notes: $N_{\text {gal }}$ is the number of galaxies in the sample. ML refers to the sample of early-type galaxies with reliable spheroid luminosity measurements. 
Table 4 | Galaxies with dynamical measurements of $M_{\mathrm{BH}}$

\begin{tabular}{|c|c|c|c|c|c|c|c|c|c|}
\hline Galaxy & Type & $\begin{array}{c}D \\
(\mathrm{Mpc}) \\
\end{array}$ & $\begin{array}{c}\sigma \\
\left(\mathrm{km} \mathrm{s}^{-1}\right)\end{array}$ & $\begin{array}{c}\log _{10}\left(L_{V, \mathrm{sph}}\right) \\
\log _{10}\left(L_{\odot}\right)\end{array}$ & $\begin{array}{l}M_{\mathrm{BH}} \\
\left(M_{\odot}\right)\end{array}$ & $\begin{array}{c}M_{\mathrm{BH}, \min } \\
\left(M_{\odot}\right)\end{array}$ & $\begin{array}{c}M_{\mathrm{BH}, \max } \\
\left(M_{\odot}\right)\end{array}$ & Method & Ref. \\
\hline Milky Way $^{a}$ & Sbc & 0.008 & $103 \pm 20$ & & $4.1 \times 10^{6}$ & $3.5 \times 10^{6}$ & $4.7 \times 10^{6}$ & stars & 941,42 \\
\hline A1836-BCG & $\mathrm{E}$ & 157.5 & $288 \pm 14$ & 11.26 & $3.9 \times 10^{9}$ & $3.3 \times 10^{9}$ & $4.3 \times 10^{9}$ & gas & 9,15 \\
\hline $\mathrm{A} 3565-\mathrm{BCG}^{b}$ & $\mathrm{E}$ & 54.4 & $322 \pm 16$ & 11.24 & $1.4 \times 10^{9}$ & $1.2 \times 10^{9}$ & $1.7 \times 10^{9}$ & gas & 15 \\
\hline Circinus & $\mathrm{Sb}$ & 4.0 & $158 \pm 18$ & & $1.7 \times 10^{6}$ & $1.4 \times 10^{6}$ & $2.1 \times 10^{6}$ & masers & 9.43 \\
\hline $\mathrm{IC} 1459^{c}$ & $\mathrm{E} 4$ & 30.9 & $315 \pm 16$ & 10.96 & $2.8 \times 10^{9}$ & $1.6 \times 10^{9}$ & $3.9 \times 10^{9}$ & stars & 9.44 \\
\hline N221 (M32) & E2 & 0.86 & $75 \pm 3$ & 8.66 & $3.1 \times 10^{6}$ & $2.5 \times 10^{6}$ & $3.7 \times 10^{6}$ & stars & 9.45 \\
\hline N224 (M31) & $\mathrm{Sb}$ & 0.80 & $160 \pm 8$ & & $1.5 \times 10^{8}$ & $1.2 \times 10^{8}$ & $2.4 \times 10^{8}$ & stars & 9,46 \\
\hline N524 & So & 23.3 & $235 \pm 12$ & 10.67 & $8.3 \times 10^{8}$ & $7.9 \times 10^{8}$ & $9.2 \times 10^{8}$ & stars & 47 \\
\hline N821 & $\mathrm{E} 4$ & 25.5 & $209 \pm 10$ & 10.43 & $1.8 \times 10^{8}$ & $1.0 \times 10^{8}$ & $2.6 \times 10^{8}$ & stars & 38 \\
\hline $\mathrm{N} 1023$ & SB0 & 12.1 & $205 \pm 10$ & 10.18 & $14.6 \times 10^{7}$ & $4.1 \times 10^{7}$ & $5.1 \times 10^{7}$ & stars & 9.48 \\
\hline N1068 (M77) & $\mathrm{SB}$ & 15.4 & $151 \pm 7$ & & $8.6 \times 10^{6}$ & $8.3 \times 10^{6}$ & $8.9 \times 10^{6}$ & masers & 9,49 \\
\hline $\mathrm{N} 1194^{b, d}$ & $\mathrm{SA} 0+$ & 55.5 & $148_{-22}^{+26}$ & & $6.8 \times 10^{7}$ & $6.5 \times 10^{7}$ & $7.1 \times 10^{7}$ & masers & 35 \\
\hline N1300 & $\mathrm{SB}(\mathrm{rs}) \mathrm{bc}$ & 20.1 & $218 \pm 10$ & & $7.1 \times 10^{7}$ & $3.6 \times 10^{7}$ & $1.4 \times 10^{8}$ & gas & 9.50 \\
\hline N1316 & E & 18.6 & $226 \pm 11$ & 11.26 & $1.5 \times 10^{8}$ & $1.24 \times 10^{8}$ & $1.75 \times 10^{8}$ & stars & 17 \\
\hline N1332 & So & 22.3 & $328 \pm 16$ & 10.14 & $1.45 \times 10^{9}$ & $1.25 \times 10^{9}$ & $1.65 \times 10^{9}$ & stars & 51 \\
\hline $\mathrm{N} 1399^{e, f}$ & E1 & 21.1 & $296 \pm 15$ & 10.78 & $5.1 \times 10^{8}$ & $4.4 \times 10^{8}$ & $5.8 \times 10^{8}$ & stars & 9.52 \\
\hline $\mathrm{N} 1399^{e, f}$ & E1 & 21.1 & $296 \pm 15$ & 10.78 & $1.3 \times 10^{9}$ & $6.4 \times 10^{8}$ & $1.8 \times 10^{9}$ & stars & 9.53 \\
\hline $\mathrm{N} 2273^{b, d}$ & $\mathrm{SB}(\mathrm{r}) \mathrm{a}$ & 26.8 & $144_{-16}^{+18}$ & & $7.8 \times 10^{6}$ & $7.4 \times 10^{6}$ & $8.2 \times 10^{6}$ & masers & 35 \\
\hline N2549 & So & 12.3 & $145 \pm 7$ & 9.60 & $1.4 \times 10^{7}$ & $1.0 \times 10^{7}$ & $1.47 \times 10^{7}$ & stars & 47 \\
\hline $\mathrm{N} 2748$ & $\mathrm{Sc}$ & 24.9 & $115 \pm 5$ & & $4.7 \times 10^{7}$ & $8.6 \times 10^{6}$ & $8.5 \times 10^{7}$ & gas & 9.50 \\
\hline N2787 & SB0 & 7.9 & $189 \pm 9$ & & $4.3 \times 10^{7}$ & $3.8 \times 10^{7}$ & $4.7 \times 10^{7}$ & gas & 9.54 \\
\hline $\mathrm{N} 2960^{b, d}$ & $\mathrm{Sa}$ & 75.3 & $166_{-15}^{+16}$ & & $1.21 \times 10^{7}$ & $1.16 \times 10^{7}$ & $1.26 \times 10^{7}$ & masers & 35 \\
\hline N3031 (M81) & $\mathrm{Sb}$ & 4.1 & $143 \pm 7$ & & $8.0 \times 10^{7}$ & $6.9 \times 10^{7}$ & $1.0 \times 10^{8}$ & gas & 9.55 \\
\hline N3115 & So & 10.2 & $230 \pm 11$ & 10.40 & $9.6 \times 10^{8}$ & $6.7 \times 10^{8}$ & $1.5 \times 10^{9}$ & stars & 9.56 \\
\hline N3227 & $\mathrm{SBa}$ & 17.0 & $133 \pm 12$ & & $1.5 \times 10^{7}$ & $7.0 \times 10^{6}$ & $2.0 \times 10^{7}$ & stars & $9[57$ \\
\hline N3245 & S0 & 22.1 & $205 \pm 10$ & & $2.2 \times 10^{8}$ & $1.7 \times 10^{8}$ & $2.7 \times 10^{8}$ & gas & 9.58 \\
\hline $\mathrm{N} 3368^{g}$ & $\mathrm{SAB}(\mathrm{rs}) \mathrm{ab}$ & 10.4 & $122_{-24}^{+28}$ & & $7.5 \times 10^{6}$ & $6.0 \times 10^{6}$ & $9.0 \times 10^{6}$ & stars & 59 \\
\hline N3377 & E6 & 11.7 & $145 \pm 7$ & 9.98 & $1.9 \times 10^{8}$ & $9.0 \times 10^{7}$ & $2.9 \times 10^{8}$ & stars & 38 \\
\hline $\mathrm{N} 3379(\mathrm{M} 105)^{b}$ & E0 & 11.7 & $206 \pm 10$ & 10.37 & $4.6 \times 10^{8}$ & $3.4 \times 10^{8}$ & $5.7 \times 10^{8}$ & stars & 33 \\
\hline N3384 & E1 & 11.7 & $143 \pm 7$ & 9.90 & $1.1 \times 10^{7}$ & $6.0 \times 10^{6}$ & $1.6 \times 10^{7}$ & stars & 38 \\
\hline $\mathrm{N} 3393^{b}$ & $\mathrm{SB}(\mathrm{rs})$ & 53.6 & $148 \pm 10$ & & $3.3 \times 10^{7}$ & $3.1 \times 10^{7}$ & $3.5 \times 10^{7}$ & masers & 60 \\
\hline $\mathrm{N} 3489^{g}$ & $\mathrm{SAB}(\mathrm{rs}) 0+$ & 12.1 & $100_{-11}^{+15}$ & & $6.0 \times 10^{6}$ & $5.2 \times 10^{6}$ & $6.8 \times 10^{6}$ & stars & 59 \\
\hline N3585 & S0 & 21.2 & $213 \pm 10$ & 10.65 & $3.4 \times 10^{8}$ & $2.8 \times 10^{8}$ & $4.9 \times 10^{8}$ & stars & 9,61 \\
\hline $\mathrm{N} 3607^{h}$ & E1 & 19.9 & $229 \pm 11$ & & $1.2 \times 10^{8}$ & $7.9 \times 10^{7}$ & $1.6 \times 10^{8}$ & stars & 9.61 \\
\hline N3608 & E1 & 23.0 & $182 \pm 9$ & 10.35 & $4.7 \times 10^{8}$ & $3.7 \times 10^{8}$ & $5.7 \times 10^{8}$ & stars & 38 \\
\hline $\mathrm{N} 3842^{i}$ & $\mathrm{E}$ & 98.4 & $270 \pm 14$ & 11.20 & $9.7 \times 10^{9}$ & $7.2 \times 10^{9}$ & $1.27 \times 10^{10}$ & stars & \\
\hline N3998 & So & 14.9 & $305 \pm 15$ & & $2.4 \times 10^{8}$ & $6.2 \times 10^{7}$ & $4.5 \times 10^{8}$ & gas & 9,62 \\
\hline N4026 & S0 & 15.6 & $180 \pm 9$ & 9.86 & $2.1 \times 10^{8}$ & $1.7 \times 10^{8}$ & $2.8 \times 10^{8}$ & stars & 9,61 \\
\hline N4258 & $\mathrm{SABbc}$ & 7.2 & $115 \pm 10$ & & $3.78 \times 10^{7}$ & $3.77 \times 10^{7}$ & $3.79 \times 10^{7}$ & masers & 9.63 \\
\hline N4261 & E2 & 33.4 & $315 \pm 15$ & 11.02 & $5.5 \times 10^{8}$ & $4.3 \times 10^{8}$ & $6.6 \times 10^{8}$ & gas & 9.64 \\
\hline N4291 & $\mathrm{E} 2$ & 25.0 & $242 \pm 12$ & 10.20 & $9.2 \times 10^{8}$ & $6.3 \times 10^{8}$ & $1.21 \times 10^{9}$ & stars & 38 \\
\hline $\mathrm{N} 4342$ & So & 18.0 & $225 \pm 11$ & & $3.6 \times 10^{8}$ & $2.4 \times 10^{8}$ & $5.6 \times 10^{8}$ & stars & 9,65 \\
\hline N4374 (M84) & E1 & 17.0 & $296 \pm 14$ & 10.91 & $8.5 \times 10^{8}$ & $7.7 \times 10^{8}$ & $9.4 \times 10^{8}$ & gas & 66 \\
\hline $\mathrm{N} 4388^{b, d}$ & $\mathrm{SA}(\mathrm{s}) \mathrm{b}$ & 19.8 & $107_{-7}^{+8}$ & & $8.8 \times 10^{6}$ & $8.6 \times 10^{6}$ & $9.0 \times 10^{6}$ & masers & 35 \\
\hline N4459 & E2 & 17.0 & $167 \pm 8$ & 10.36 & $7.4 \times 10^{7}$ & $6.0 \times 10^{7}$ & $8.8 \times 10^{7}$ & gas & 9.54 \\
\hline $\mathrm{N} 4473$ & $\mathrm{E} 4$ & 17.0 & $190 \pm 9$ & 10.39 & $1.0 \times 10^{8}$ & $5.0 \times 10^{7}$ & $1.5 \times 10^{8}$ & stars & 38 \\
\hline $\mathrm{N} 4486(\mathrm{M} 87)^{b, j}$ & E1 & 17.0 & $324_{-16}^{+28}$ & 11.10 & $6.3 \times 10^{9}$ & $5.9 \times 10^{9}$ & $6.6 \times 10^{9}$ & stars & 4 \\
\hline $\mathrm{N} 4486 \mathrm{~A}$ & $\mathrm{E} 2$ & 17.0 & $111 \pm 5$ & 9.41 & $1.3 \times 10^{7}$ & $9.0 \times 10^{6}$ & $1.8 \times 10^{7}$ & stars & 9,67 \\
\hline $\mathrm{N} 4564^{k}$ & So & 17.0 & $162 \pm 8$ & & $9.4 \times 10^{7}$ & $6.8 \times 10^{7}$ & $1.2 \times 10^{8}$ & stars & 38 \\
\hline N4594 (M104) & $\mathrm{Sa}$ & 10.3 & $240 \pm 12$ & & $5.3 \times 10^{8}$ & $4.74 \times 10^{8}$ & $6.08 \times 10^{8}$ & stars & 36 \\
\hline
\end{tabular}


Table 4, continued

\begin{tabular}{|c|c|c|c|c|c|c|c|c|c|}
\hline Galaxy & Type & $\begin{array}{c}D \\
(\mathrm{Mpc}) \\
\end{array}$ & $\begin{array}{c}\sigma \\
\left(\mathrm{km} \mathrm{s}^{-1}\right) \\
\end{array}$ & $\begin{array}{c}\log _{10}\left(L_{V, \mathrm{sph}}\right) \\
\log _{10}\left(L_{\odot}\right) \\
\end{array}$ & $\begin{array}{l}M_{\mathrm{BH}} \\
\left(M_{\odot}\right) \\
\end{array}$ & $\begin{array}{c}M_{\mathrm{BH}, \min } \\
\left(M_{\odot}\right)\end{array}$ & $\begin{array}{c}M_{\mathrm{BH}, \max } \\
\left(M_{\odot}\right) \\
\end{array}$ & Method & Ref. \\
\hline N4596 & SB0 & 18.0 & $136 \pm 6$ & & $8.4 \times 10^{7}$ & $5.9 \times 10^{7}$ & $1.2 \times 10^{8}$ & gas & 954 \\
\hline $\mathrm{N} 4649(\mathrm{M} 60)^{b, l}$ & E2 & 16.5 & $341 \pm 17$ & 10.99 & $4.7 \times 10^{9}$ & $3.7 \times 10^{9}$ & $5.8 \times 10^{9}$ & stars & 37 \\
\hline N4697 & E6 & 12.4 & $177 \pm 8$ & 10.45 & $2.0 \times 10^{8}$ & $1.8 \times 10^{8}$ & $2.2 \times 10^{8}$ & stars & 38 \\
\hline N4736 (M94) & Sab & 4.9 & $112 \pm 6$ & & $6.68 \times 10^{6}$ & $5.14 \times 10^{6}$ & $8.22 \times 10^{6}$ & stars & 36 \\
\hline N4826 (M64) & Sab & 6.4 & $96 \pm 5$ & & $1.36 \times 10^{6}$ & $1.02 \times 10^{6}$ & $1.71 \times 10^{6}$ & stars & 36 \\
\hline $\mathrm{N} 4889^{m}$ & $\mathrm{E}$ & 103.2 & $347 \pm 17$ & 11.42 & $2.1 \times 10^{10}$ & $5.5 \times 10^{9}$ & $3.7 \times 10^{10}$ & stars & \\
\hline N5077 & E3 & 44.9 & $222 \pm 11$ & 10.75 & $8.0 \times 10^{8}$ & $4.7 \times 10^{8}$ & $1.3 \times 10^{9}$ & gas & 968 \\
\hline $\mathrm{N} 5128^{e}$ & S0/E & 4.4 & $150 \pm 7$ & 10.66 & $3.0 \times 10^{8}$ & $2.8 \times 10^{8}$ & $3.4 \times 10^{8}$ & stars & 969 \\
\hline $\mathrm{N} 5128^{e}$ & S0/E & 4.4 & $150 \pm 7$ & 10.66 & $7.0 \times 10^{7}$ & $3.2 \times 10^{7}$ & $8.3 \times 10^{7}$ & stars & 970 \\
\hline N5576 & E3 & 27.1 & $183 \pm 9$ & 10.44 & $1.8 \times 10^{8}$ & $1.4 \times 10^{8}$ & $2.1 \times 10^{8}$ & stars & 961 \\
\hline N5845 & E3 & 28.7 & $234 \pm 11$ & 9.84 & $5.4 \times 10^{8}$ & $3.7 \times 10^{8}$ & $7.1 \times 10^{8}$ & stars & 38 \\
\hline N6086 ${ }^{b}$ & $\mathrm{E}$ & 139.1 & $318 \pm 16$ & 11.18 & $3.8 \times 10^{9}$ & $2.6 \times 10^{9}$ & $5.5 \times 10^{9}$ & stars & 16 \\
\hline N6251 & E1 & 106.0 & $290 \pm 14$ & & $6.0 \times 10^{8}$ & $4.0 \times 10^{8}$ & $8.0 \times 10^{8}$ & gas & 971 \\
\hline $\mathrm{N} 6264^{b, d}$ & $\mathrm{~S}$ & 145.4 & $158_{-14}^{+15}$ & & $3.03 \times 10^{7}$ & $2.99 \times 10^{7}$ & $3.08 \times 10^{7}$ & masers & 35 \\
\hline $\mathrm{N} 6323^{b, d}$ & Sab & 110.5 & $158_{-23}^{+28}$ & & $9.8 \times 10^{6}$ & $9.7 \times 10^{6}$ & $9.9 \times 10^{6}$ & masers & 35 \\
\hline N7052 & E3 & 70.9 & $266 \pm 13$ & 10.87 & $4.0 \times 10^{8}$ & $2.4 \times 10^{8}$ & $6.8 \times 10^{8}$ & gas & 9172 \\
\hline N7457 & So & 14.0 & $67 \pm 3$ & 9.42 & $1.0 \times 10^{7}$ & $4.0 \times 10^{6}$ & $1.6 \times 10^{7}$ & stars & 38 \\
\hline N7582 & SBab & 22.3 & $156 \pm 19$ & & $5.5 \times 10^{7}$ & $4.4 \times 10^{7}$ & $7.1 \times 10^{7}$ & gas & 973 \\
\hline $\mathrm{U} 3789^{b, d}$ & $\mathrm{SA}(\mathrm{r}) \mathrm{ab}$ & 48.4 & $107_{-12}^{+13}$ & & $1.08 \times 10^{7}$ & $1.03 \times 10^{7}$ & $1.14 \times 10^{7}$ & masers & 35 \\
\hline
\end{tabular}

Notes: $D$ is the distance to the galaxy, assuming $H_{0}=70 \mathrm{~km} \mathrm{~s}^{-1} \mathrm{Mpc}^{-1} . L_{V \text {,sph }}$ is the $V$-band stellar luminosity of the galaxy's spheroidal component. $M_{\mathrm{BH}, \min }$ and $M_{\mathrm{BH}, \max }$ are the upper and lower bounds of the $68 \%$ confidence interval in $M_{\mathrm{BH}}$.

${ }^{a}$ The literature contains a large number of estimates for the velocity dispersion of our Galaxy's bulge, using different kinematic tracers at different radii. We use the radially averaged measurement of $\sigma=103 \pm 20 \mathrm{~km} \mathrm{~s}^{-1}$ from Tremaine et al. (2002) 39 .

${ }^{b} \mathrm{G} 09$ use the convention $H_{0}=70 \mathrm{~km} \mathrm{~s}^{-1} \mathrm{Mpc}^{-1}$ for all galaxies whose distances are derived from systemic velocity measurements. To match this convention, we have adjusted the distance to several galaxies in our updated sample. All distance adjustments yield corresponding adjustments to $M_{\mathrm{BH}}, M_{\mathrm{BH}, \min }$, and $M_{\mathrm{BH}}$,max , such that $M_{\mathrm{BH}} \propto D$. Our reported $68 \%$ confidence intervals for $M_{\mathrm{BH}}$ do not include uncertainties in the distance measurements.

${ }^{c}$ We derive an effective velocity dispersion of $315 \mathrm{~km} \mathrm{~s}^{-1}$ for IC 1459, using spatially resolved major-axis kinematics from Cappellari et al. (2002) ${ }^{44}$, at radii of $0.8-25.1$ arcseconds. For $r<0.8$ arcseconds, stellar motions are directly influenced by the central black hole.

${ }^{d}$ Maser-based black hole masses for several galaxies are presented in Greene et al. (2010) ${ }^{34}$ and Kuo et al. (2011) ${ }^{35}$. We use the velocity dispersions presented in Greene et al. (2010). For consistency with the rest of our sample, we use the black hole masses from Kuo et al. (2011), which agree with the values in Greene et al. (2010) but do not include distance uncertainties in the overall uncertainty for $M_{\mathrm{BH}}$. Braatz et al. (2010) ${ }^{74}$ provide an updated distance and black hole mass for UGC 3789, which are consistent with the values we adopt from Kuo et al. (2011).

${ }^{e}$ Following G09, our sample includes two distinct measurements for NGC 1399 and also for NGC 5128. We weight each of these measurements by $50 \%$ when performing fits to $M_{\mathrm{BH}}(\sigma)$ and $M_{\mathrm{BH}}(L)$. In Figure 3 we only plot the more recent measurement for each galaxy.

${ }^{f}$ We derive an effective velocity dispersion of $296 \mathrm{~km} \mathrm{~s}^{-1}$ for NGC 1399, using spatially resolved measurements from Graham et al. (1998) ${ }^{75}$ and Gebhardt et al. (2007) ${ }^{[52}$ at radii of $0.6-41$ arcseconds. For $r<0.6$ arcseconds, stellar motions are directly influenced by the central black hole.

${ }^{g}$ NGC 3368 and NGC 3489 were not included in our sample until late in the editorial process, and so do not contribute toward the fits reported in the main letter or in Table 3. Still, we view the reported black hole masses for NGC 3368 and NGC 3489 as valid measurements that should be included in future studies. For the velocity dispersions, we use the average values and outer limits of the various measurements reported by Nowak et al. (2010) 59. Including NGC 3368 and NGC 3489, the $M_{\mathrm{BH}}-\sigma$ parameters $\left(\alpha, \beta, \epsilon_{0}\right)$ are $(8.28 \pm 0.06,5.13 \pm 0.34,0.42)$ for 67 galaxies, $(8.38 \pm 0.06$, $4.57 \pm 0.36,0.36)$ for 46 early-type galaxies, and $(8.00 \pm 0.21,4.76 \pm 1.15,0.43)$ for 21 late-type galaxies. These parameters are consistent with our reported values for the sample of 65 galaxies.

${ }^{h}$ The literature contains two inconsistent estimates of the stellar luminosity in NGC 3607: $M_{V}=-21.62$ in G09, and $M_{V}=-19.88$ in Lauer et al. $(2007)^{14}$.

${ }^{i}$ We derive an effective velocity dispersion of $270 \mathrm{~km} \mathrm{~s}^{-1}$ for NGC 3842 , using measurements from GMOS and VIRUS-P at radii of 1.2 - 29.8 arcseconds. For $r<1.2$ arcseconds, stellar motions are directly influenced by the central black hole.

${ }^{j}$ For M87, we use the updated velocity dispersion of $324 \mathrm{~km} \mathrm{~s}^{-1}$ from Gebhardt et al. (2011) ${ }^{4]}$, based on measurements at radii of $2.1-100$ arcseconds. For $r<2.1$ arcseconds, stellar motions are directly influenced by the central black hole.

${ }^{k}$ The literature contains two inconsistent estimates of the bulge stellar luminosity in NGC 4564: $M_{V}=-19.60$ in G09, and $M_{V}=-20.26$ in Lauer et al. (2007) 14 .

${ }^{l}$ We derive an effective velocity dispersion of $341 \mathrm{~km} \mathrm{~s}^{-1}$ for M60, using spatially resolved measurements from Pinkney et al. (2003) ${ }^{27}$ at radii of 2.2 - 44 arcseconds. For $r<2.2$ arcseconds, stellar motions are directly influenced by the central black hole.

${ }^{m}$ Our quoted value of $M_{\mathrm{BH}}$ for NGC 4889 is the median of the $68 \%$ confidence interval $0.7-3.4 \times 10^{10} M_{\odot}$. We derive an effective velocity dispersion of $347 \mathrm{~km} \mathrm{~s}^{-1}$, using kinematics from Loubser et al. (2008) $\stackrel{13}{13}$ at radii of $2.0-22.9$ arcseconds. The inner cutoff of 2.0 arcseconds excludes the asymmetric velocity dispersion peak and possible stellar torus. 